DRAFT VERSION MARCH 18, 2021

Typeset using LATEX twocolumn style in AASTeX63

\title{
ALMA observation of the protoplanetary disk around WW Cha: faint double-peaked ring and asymmetric structure
}

\author{
Kazuhiro D. Kanagawa,,${ }^{1,2}$ Jun Hashimoto, ${ }^{3}$ Takayuki Muto, ${ }^{4}$ TaKashi Tsukagoshi, ${ }^{5}$ Sanemichi Z. TAKahashi, ${ }^{5}$ \\ Yasuhiro Hasegawa, ${ }^{6}$ Mihoko Konishi, ${ }^{7}$ Hideko Nomura, ${ }^{5}$ Hauyu Baobab Liu, ${ }^{8}$ Ruobing Dong, ${ }^{9}$ Akimasa Kataoka, ${ }^{5}$ \\ Munetake Momose, ${ }^{2}$ Tomohiro Ono, ${ }^{10}$ Michael Sitko, ${ }^{11,12}$ Michihiro Takami, ${ }^{8}$ And Kengo Tomida ${ }^{13}$ \\ ${ }^{1}$ Research Center for the Early Universe, Graduate School of Science, The University of Tokyo, Hongo, Bunkyo-ku, Tokyo 113-0033, Japan \\ ${ }^{2}$ College of Science, Ibaraki University, 2-1-1 Bunkyo, Mito, Ibaraki 310-8512, Japan \\ ${ }^{3}$ Astrobiology Center, National Institutes of Natural Sciences, 2-21-1 Osawa, Mitaka, Tokyo 181-8588, Japan \\ ${ }^{4}$ Division of Liberal Arts, Kogakuin University, 1-24-2 Nishi-Shinjuku, Shinjuku-ku, Tokyo 163-8677, Japan \\ ${ }^{5}$ National Astronomical Observatory of Japan, 2-21-1 Osawa, Mitaka, Tokyo 181-8588, Japan \\ ${ }^{6}$ Jet Propulsion Laboratory, California Institute of Technology, Pasadena, CA 91109, USA \\ ${ }^{7}$ Faculty of Science and Technology, Oita University, 700 Dannoharu, Oita 870-1192, Japan \\ ${ }^{8}$ Institute of Astronomy and Astrophysics, Academia Sinica, $11 \mathrm{~F}$ of Astronomy-Mathematics Building, AS No.1, Sec. 4, Roosevelt Rd, Taipei 10617, Taiwan, \\ R.O.C. \\ ${ }^{9}$ Department of Physics \& Astronomy, University of Victoria, Victoria, BC, V8P 1A1, Canada \\ ${ }^{10}$ Department of Earth and Planetary Sciences, Tokyo Institute of Technology, 2-12-1 Ookayama, Meguro-ku, Tokyo 152-8551, Japan \\ ${ }^{11}$ Department of Physics, University of Cincinnati, Cincinnati, $\mathrm{OH} 45221$, USA \\ ${ }^{12}$ Space Science Institute, 475 Walnut Street, Suite 205, Boulder, CO 80301, USA \\ ${ }^{13}$ Astronomical Institute, Tohoku University, Sendai 980-8578, Japan
}

(Received March 18, 2021; Revised March 18, 2021; Accepted March 18, 2021)

Submitted to ApJ

\begin{abstract}
We present Atacama Large Millimeter/submillimeter Array (ALMA) band 6 observations of dust continuum emission of the disk around WW Cha. The dust continuum image shows a smooth disk structure with a faint (low-contrast) dust ring, extending from $\sim 40$ au to $\sim 70$ au, not accompanied by any gap. We constructed the simple model to fit the visibility of the observed data by using MCMC method and found that the bump (we call the ring without the gap the bump) has two peaks at 40 au and 70 au. The residual map between the model and observation indicates asymmetric structures at the center and the outer region of the disk. These asymmetric structures are also confirmed by model-independent analysis of the imaginary part of the visibility. The asymmetric structure at the outer region is consistent with a spiral observed by SPHERE. To constrain physical quantities of the disk (dust density and temperature), we carried out radiative transfer simulations. We found that the midplane temperature around the outer peak is close to the freezeout temperature of $\mathrm{CO}$ on water ice $(\sim 30 \mathrm{~K})$. The temperature around the inner peak is about $50 \mathrm{~K}$, which is close to the freezeout temperature of $\mathrm{H}_{2} \mathrm{~S}$ and also close to the sintering temperature of several species. We also discuss the size distribution of the dust grains using the spectral index map obtained within the band 6 data.
\end{abstract}

Keywords: protoplanetary disks - stars:individual (WW Cha) - stars:pre-main sequence - techniques:interferometric

\section{INTRODUCTION}

Planets are born in a protoplanetary disk around a young star. Recent observations have revealed substruc-

Corresponding author: Kazuhiro D. Kanagawa

kazuhiro.kanagawa.d@vc.ibaraki.ac.jp tures such as gaps, rings, and crescents in the protoplanetary disks (e.g., Fukagawa et al. 2013; Akiyama et al. 2015, 2016; ALMA Partnership et al. 2015; Momose et al. 2015; Dong et al. 2018b; Long et al. 2018; van der Marel et al. 2019; Soon et al. 2019; Kim et al. 2020). These structure could be formed at an edge of a gap induced by disk-planet interaction (e.g., Paardekooper \& Mellema 2004; Muto \& Inutsuka 2009; Zhu et al. 2012; Dong et al. 
2015; Pinilla et al. 2015; Kanagawa et al. 2018). Alternatively, these could be associated to dust growth related to snowline (e.g., Zhang et al. 2015; Cieza et al. 2017; Macías et al. 2017; van der Marel et al. 2018; Facchini et al. 2020) and the sintering effect (Okuzumi et al. 2016), or secular gravitational instability (Takahashi \& Inutsuka 2014, 2016; Tominaga et al. 2018). The ring/gap structures of the dust grains also could be formed by axisymmetric gas perturbation due to evolution of luminosity of the central star (Vorobyov et al. 2020). In any case, these substructures could be reflected by planet formation and growth of dust grains which are building blocks of planets. Direct observations of the disks help to understand how formation of the planets progresses in the disk.

Our target, WW Cha is a young star with a circumstellar disk (e.g., Pascucci et al. 2016; Garufi et al. 2020) in the Chameleon I star-forming region. The star is located at about 190 pc (Gaia Collaboration et al. 2018, 2020). The mass of the star is about $1 M_{\odot}$, the surface temperature is $4350 \mathrm{~K}$ (Spectral type is K5) (Luhman 2007), and the luminosity is $11 L_{\odot}$ (Garufi et al. 2020). The star is very young ( $\sim 0.2 \mathrm{Myr}$ ) and it could be still embedded into the molecular cloud core with a high extinction (Ribas et al. 2013; Garufi et al. 2020). A high accretion rate onto the star, $10^{-6.6} M_{\odot} / \mathrm{yr}$, is inferred from the photometric and the Balmer continuum observations (Manara et al. 2016). Moreover, the binary with the separation of $\sim 1$ au is reported by VLTI (Anthonioz et al. 2015). The disk of WW Cha may be a pre-transition disk because strong infrared emission is detected (Espaillat et al. 2011; Ribas et al. 2013), while the recent modeling using radiative transfer simulations done by van der Marel et al. (2016) suggested an inner cavity with the radius of $\sim 50$ au (but with a large uncertainty). The disk is very bright in millimeter wavelength (Pascucci et al. 2016) and Lommen et al. (2009) reported the emission at $\sim 1 \mathrm{~cm}$, which indicates the presence of large grains due to growth of dust grains.

In this paper, we report dust continuum observations of the disk around WW Cha in ALMA Cycle 5. In Section 2, we describe the setup of the observation and show the observational results. We developed a model for the observed emission by using the Markov Chain Monte Carlo (MCMC) method and found axisymmetric substructures and asymmetric structures, which is described in Section 3. Moreover, we carried out radiative transfer simulations to constrain the physical parameters of the disk which are described in Section 4. In Section 5, we discuss origins of the substructures, an inner cavity and binary, and the dust growth in the disk. Section 6 contains our conclusion.

\section{OBSERVATIONS AND RESULTS}

The observation was carried out by ALMA in band 6, which is summarized in Table 1. The data were calibrated by the Common Astronomy Software Applications (CASA) package (McMullin et al. 2007) version 5.6.1-8, following the calibration scripts provided by ALMA. We conducted self-calibration of the visibilities. The phases were self-calibrated once with a fairly long solution intervals (solint='inf') combining all spectral windows (SPWs).

We combined the data taken by sparse (C43-8) and compact (C43-4) array configurations to recover the missing flux at larger angular scales. By using the CASA tool uvmodelfit, we fitted the data by a Gaussian shape, and the phase center was corrected to be the center of the Gaussian shape by fixvis. The inclination $i=37.2^{\circ} \pm$ $0.026^{\circ}$ and position angle $\phi=32.4^{\circ} \pm 0.04^{\circ}$ are obtained by the Gaussian fit by uvmodelfit ${ }^{1}$. There are two SPWs for continuum with $1.875 \mathrm{GHz}$ frequency width with the central frequency being $233.0 \mathrm{GHz}$ (Upper band) and $216.7 \mathrm{GHz}$ (Lower band) in both the C43-4 and C43-8 data. As shown below, the total flux density of the data in $233.0 \mathrm{GHz}$ ( 500 mJy) is significantly larger than that in $216.7 \mathrm{GHz}$ ( $430 \mathrm{mJy})$. Hence, the dust continuum image of combined data was synthesized by CASA with the tclean task using the mtmfs algorithm (Rau \& Cornwell 2011) with nterms $=2$. We obtained a synthesized image at $224.9 \mathrm{GHz}$ with the beam size of $89.6 \times 60.0$ mas $(17.0 \times 11.4 \mathrm{au})$ with $\mathrm{PA}=168.8^{\circ}$ and with the $1 \sigma \mathrm{RMS}$ noise level of $0.029 \mathrm{mJy} /$ Beam. The imaging parameters are summarized in Table 1.

The synthesized image is shown in Figure 1. The panel (a) shows the synthesized dust continuum image derived from all SPW data, and the brightness temperatures along the major and minor axes are shown in the panel (b). The dust disk is clearly resolved and its size is about 0.5 arcsec, which corresponds to about 100 au. We see a faint low-contrast dust ring feature in the radial profile of brightness temperature at $\sim 0.3 \operatorname{arcsec}(\sim 60$ au) from the central star. This ring structure is not accompanied with the gap structure, as different from the rings found in the disk of HL Tau (ALMA Partnership et al. 2015), and DSHARP's samples (Andrews et al. 2018). Hence, we call this ring structure (without the gap) the bump in the following. The total flux density with $>3 \sigma(0.087 \mathrm{mJy})$ emission is measured to be $449.58 \mathrm{mJy}$. We do not see a clear cavity structure in the image. Therefore, the large cavity such as that predicted

\footnotetext{
${ }^{1}$ The fitted inclination and position angle are slightly different in the C438 (sparse configuration) and $\mathrm{C} 43-4$ (compact configuration) data. We adopted the values of the C43-8 data. For the C43-4 data, $i=39.2^{\circ}$ and $\phi=30.9^{\circ}$ with the relatively large reduced $\chi^{2}, 5.78$ while, for the fit of the C43-8 data, reduced $\chi^{2}=1.89$. The relatively large $\chi^{2}$ for the C43-4 data can be due to the asymmetric structure discussed in Section 3.4. Hence, we adopted the values given by the fit of the C43-8 data
} 


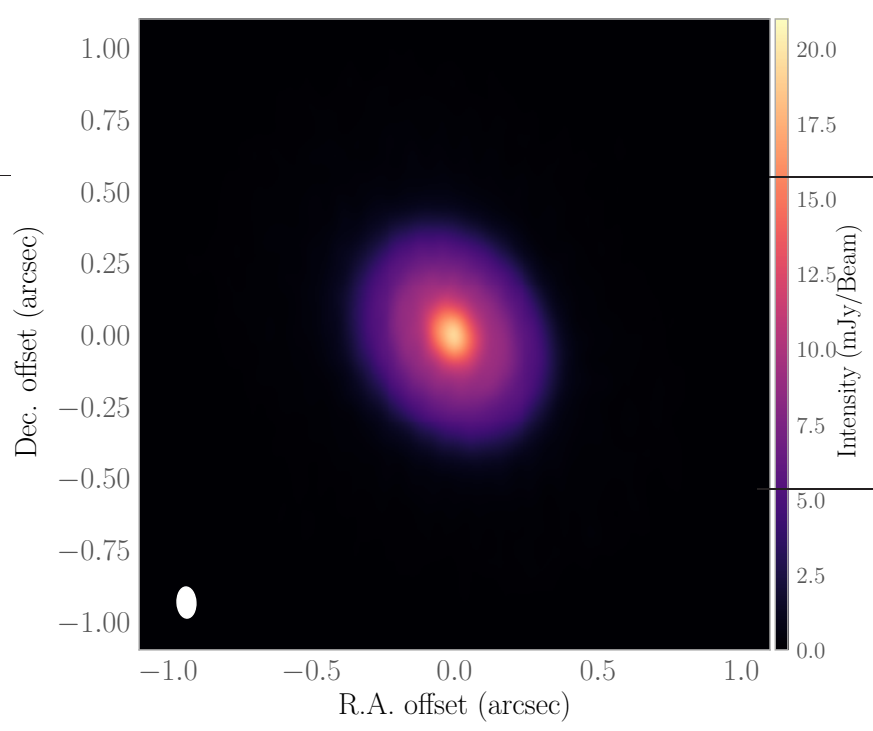

(a) Combined image at $224.9 \mathrm{GHz}$

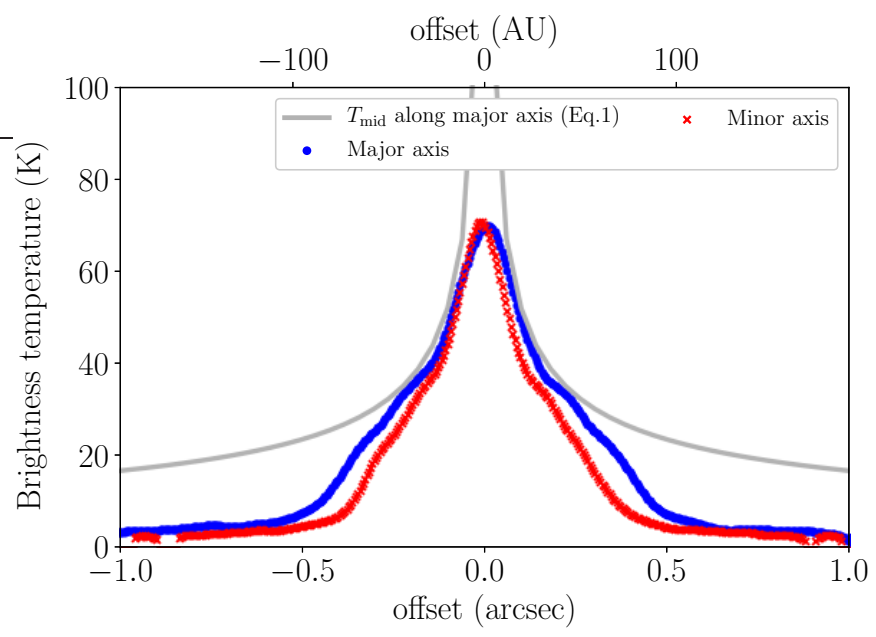

(b) Radial slices of Panel (a)

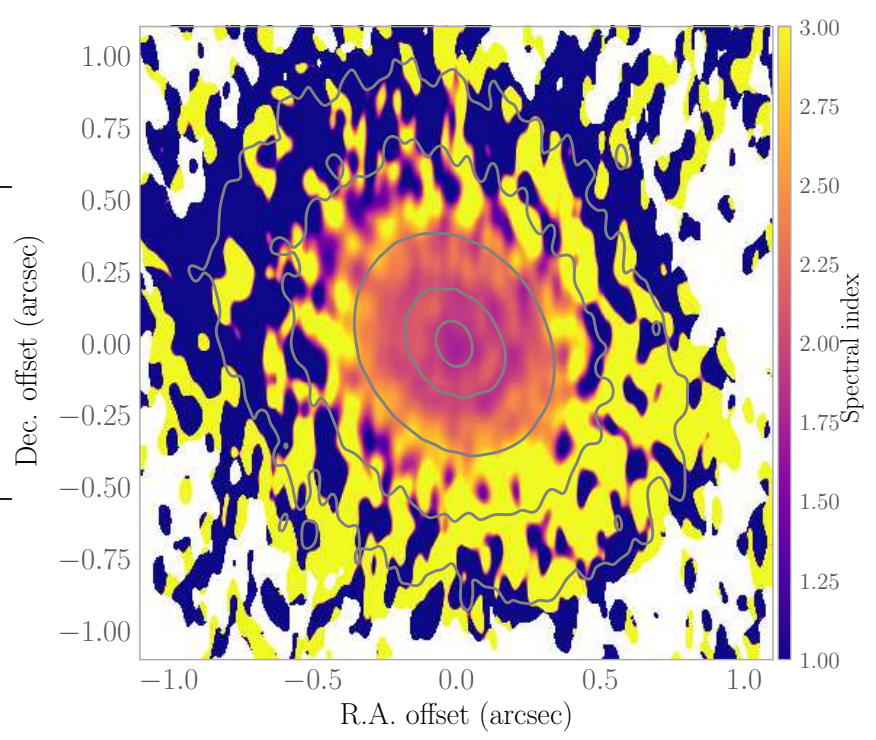

(c) Image of spectral index

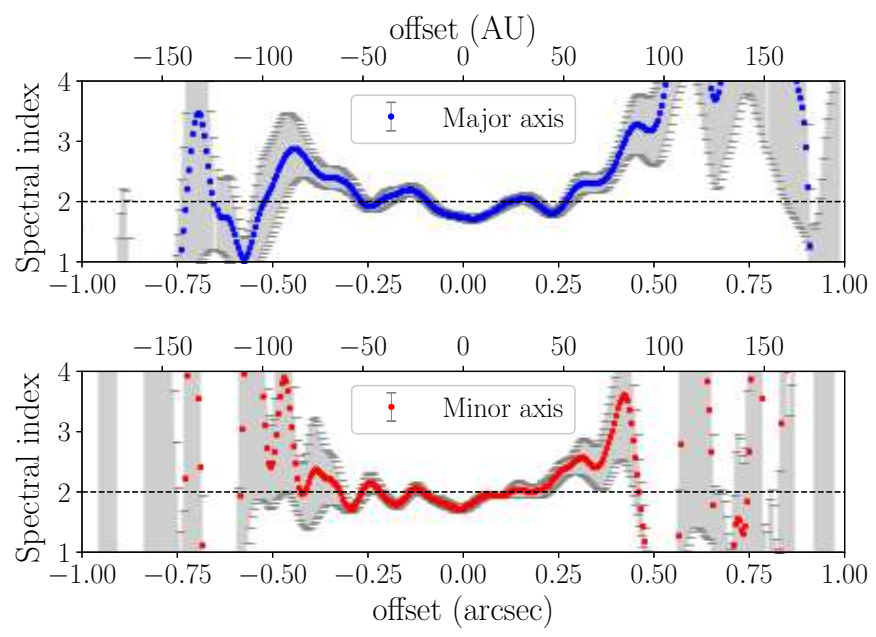

(d) Radial slice of Panel (c)

Figure 1. panels (a) and (c) show the image and the spectral index maps resulting from the combination of $233.0 \mathrm{GHz}$ and $216.7 \mathrm{GHz}$ data. The contours in Panel (c) indicate the intensity levels of $0.087 \mathrm{mJy} /$ Beam $(3 \sigma), 0.29 \mathrm{mJy} / \mathrm{Beam}(10 \sigma), 2.9 \mathrm{mJy} / \mathrm{Beam}(100 \sigma), 8.7 \mathrm{mJy} / \mathrm{Beam}$ $(300 \sigma)$ and $14.5 \mathrm{mJy} /$ Beam $(500 \sigma)$ in Panel (a). Panels (b) and (d) show brightness temperature and spectral index along the major and minor axis, respectively. The gray thick lines in Panel (b) denote the midplane temperature given by Equation (1) with $L_{*}=11 L_{\odot}$.

by SED analysis done by van der Marel et al. (2016), is ruled out at least in the millimeter image, while the existence of a cavity that is smaller than the beam size is not ruled out.

In the panel (c) of Figure 1, we show the spectral index map, and panel (d) illustrates the spectral indexes along the major and minor axes. Within the region of $<0.25$ arcsec ( $<50 \mathrm{au}$ ), the spectral index is $\sim 2$, which is an indicative of optically thick dust emission. Around the center of the disk, in particular, the spectral index is slightly below 2 , which may indicate optically thick dust scattering (Liu 2019; Zhu et al. 2019). In the region where the offset is larger than 0.25 arcsec, the disk may be optically thin because the spec- tral index is larger than 2, and the spectral index seems to increase in the outer region, though there is large uncertainly at $>0.5$ arcsec.

In the panel (b) of Figure 1, we also plot the midplane temperature along the major axis, estimated by the simple expression for a passive heated radiative disk (e.g., Chiang \& Goldreich 1997),

$$
T_{\text {mid }}=\left(\frac{\alpha_{g} L_{*}}{8 \pi R^{2} \sigma_{\mathrm{SB}}}\right)^{1 / 4},
$$

where $\sigma_{\mathrm{SB}}$ is the Stefan-Bolzmann constant, and $R$ is the distance from the star. The grazing angle $\alpha_{g}$ is set to be 0.02 
Table 1. ALMA band 6 Observations and Imaging Parameters

\begin{tabular}{lcc}
\hline \hline \multicolumn{1}{c}{ Observations } & Sparse configuration & Compact configuration \\
\hline Observing date (UT) & 2017. Nov.27 & 2018. Mar.11 \\
Configuration & C43-8 & C43-4 \\
Project code & \multicolumn{2}{c}{ 2017.1.00286.S } \\
Time on source (min) & 60.3 & 29.4 \\
Number of antennas & 47 & 42 \\
Baseline lengths & $92.1 \mathrm{~m}$ to $8.5 \mathrm{~km}$ & $15.1 \mathrm{~m}$ to $1.2 \mathrm{~km}$ \\
Baseband Freqs. (GHz) & 233.0 (Upper band), 216.7 (Lower band) \\
Channel width (GHz) & \multicolumn{3}{c}{1.87} \\
Continuum band width (GHz) & \multicolumn{3}{c}{ J.0 } \\
Bandpass calibrator & $\mathrm{J} 0635-7516$ & $\mathrm{~J} 1427-4206$ \\
Flux calibrator & $\mathrm{J} 0635-7516$ & $\mathrm{~J} 1427-4206$ \\
Phase calibrator & $\mathrm{J} 1058-8003$ & $\mathrm{~J} 1058-8003$ \\
Mean PWV (mm) & 1.5 & 0.6 \\
\hline \hline
\end{tabular}

\begin{tabular}{lc}
\multicolumn{1}{c}{ Imaging } & \\
\hline \hline Robust clean parameter & 0.0 \\
Deconvolution algorithm & mtmfs \\
Weighting & Briggs \\
nterms & 2 \\
Beam shape & $89.6 \times 60.0$ mas $(17.0 \times 11.4 \mathrm{au})$ at PA of $168.8^{\circ}$ \\
r.m.s. noise $(\mathrm{mJy} / \mathrm{beam})$ & 0.029 \\
\hline
\end{tabular}

and the stellar luminosity $L_{*}$ is $11 L_{\odot}$ in the plot. The brightness temperature is close to the midplane temperature within 0.25 arcsec from the center, which indicate the optically thick emission. The outer region $(R>0.25$ arcsec or $>50 \mathrm{au})$ can be optically thin, which is consistent with the spectral index map mentioned above.

Using the inclination and position angle, we have deprojected the face-on equivalent view, to identify substructures on the disk. In Figure 2, we show the intensity profile along the major axis and minor axis in the face-on view. In Figure 2, we put gray double-sided arrows to indicate the location of the faint low-contrast dust bump, which extends from $R \simeq 0.2 \operatorname{arcsec}(\simeq 40 \mathrm{au})$ to $R \simeq 0.4 \operatorname{arcsec}(\simeq 80 \mathrm{au})$. Moreover, one can see this bump has a double-peak feature, which is indicated by the black arrows in the figure: the inner peak locates at $|R| \simeq 0.2 \operatorname{arcsec}(40 \mathrm{au}$ ) and the outer one locates at $|R| \simeq 0.35 \operatorname{arcsec}(70 \mathrm{au}$ ), where $R$ is an offset from the center. Although the double-peak feature is not clear in the image, it is confirmed by the visibility fitting described in Section 3. Outside of $0.5 \mathrm{arcsec}$, the intensity decreases quickly, but around $|R| \sim 0.75 \operatorname{arcsec}(R \sim 150 \mathrm{au})$, the slope of the intensity becomes moderate.

Figure 3 shows the azimuthal distributions of the intensity in the face-on view. There are asymmetric structures in the azimuthal distribution at $R=0.05 \operatorname{arcsec}(\sim 10 \mathrm{au})$, and the deviation from the averaged value of the averaged value at this radius is at most about $1 \mathrm{mJy} / \mathrm{Beam}(5 \%$ of the averaged
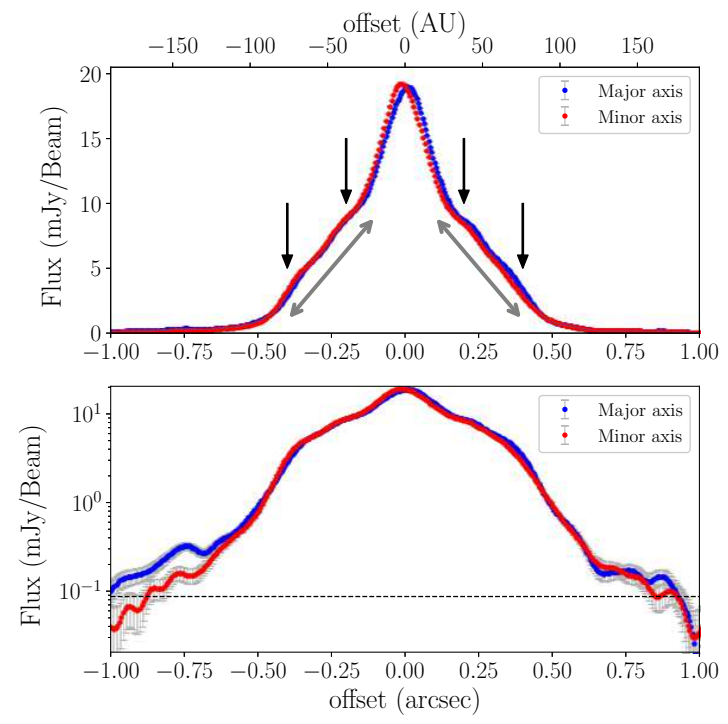

Figure 2. Intensity distributions along the major and minor axes of the face-on view of Figure 1 (a). We also plot the $1 \sigma$ noise level at each data point. The black arrows indicate the locations of the peaks within the bump structure, and the gray one indicates location of the bump structure. The dashed horizontal line denotes $3 \sigma$ noise level $(=0.087 \mathrm{mJy} / \mathrm{Beam})$. The bottom panel is the same as the top panel, but the vertical axis is in logarithmic scale. The gray double-sided arrows indicate the location of the faint dust bump and the black arrows denote the locations of the peaks within the bump.

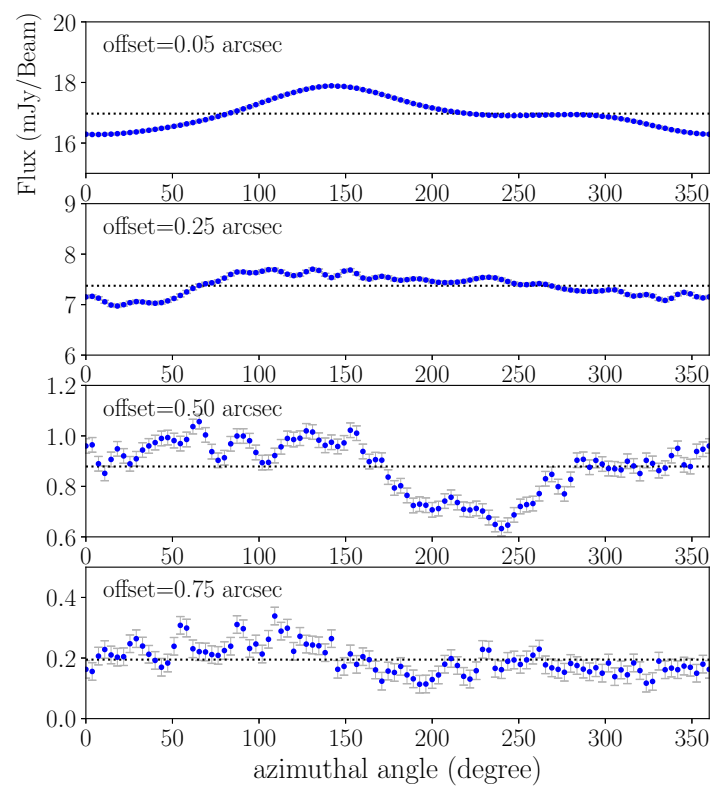

Figure 3. Azimuthal distributions of observed intensity in the faceon view, at offset $=0.05 \operatorname{arcsec}(\simeq 10 \mathrm{au}), 0.25 \operatorname{arcsec}(\simeq 50 \mathrm{au})$, $0.5 \operatorname{arcsec}(\simeq 100 \mathrm{au}), 0.75 \mathrm{arcsec}(\simeq 150 \mathrm{au})$. The error bars indicate $1 \sigma$ noise level $(0.029 \mathrm{mJy})$. The horizontal lines are the averages, which indicates $16.98 \mathrm{mJy} /$ Beam $(\sim 600 \sigma), 7.37 \mathrm{mJy} /$ Beam $(\sim 250 \sigma), 0.88 \mathrm{mJy} /$ Beam $(\sim 30 \sigma)$, and $0.20 \mathrm{mJy} /$ Beam $(\sim 7 \sigma)$, from the top panel to bottom panel. 


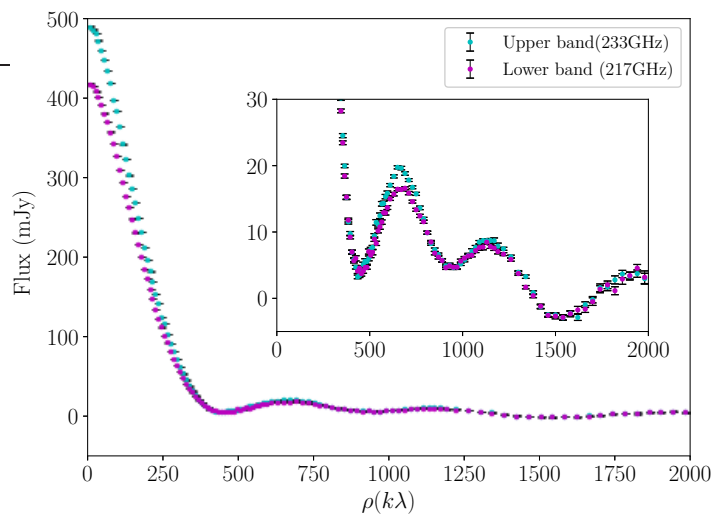

Figure 4. Real part of the visibility for the upper and lower band data. In the plot, we combine the C43-4 and C43-8 data. The inset shows the zoom in of the region with the flux $<30 \mathrm{mJy}$.

value or $\sim 30 \sigma$ ). The asymmetry at the innermost radii of the disk is also indicated in Figure 2 as the distribution along the major and minor axes do not overlap at $R<20$ mas or $<4$ au. At $R=0.25 \operatorname{arcsec}(\sim 50 \mathrm{au})$, the structure has the similar pattern of asymmetricity seen in the distribution at $R=0.05$ arcsec, and the derivation from the averaged value is about $0.3 \mathrm{mJy} / \mathrm{Beam}$ (it is about $5 \%$ of the averaged value or $\sim 10 \sigma)$. At $R=0.5 \operatorname{arcsec}(\sim 100 \mathrm{au})$, one can see a significant asymmetric structure. The intensity at $<$ $150^{\circ}$ is larger than averaged value by $0.18 \mathrm{mJy} / \mathrm{Beam}(\sim 6 \sigma)$ and it is smaller around $250^{\circ}$ by $0.25 \mathrm{mJy} /$ Beam $(\sim 9 \sigma)$. The deviation from the averaged value is about $20 \%$ of the averaged value. At $R=0.75 \operatorname{arcsec}(\sim 150 \mathrm{au})$, we may see an asymmetric structure, as the intensity at $\sim 100^{\circ}$ is larger in $0.14 \mathrm{mJy} /$ Beam $(\sim 5 \sigma)$ than the average and it is smaller than the average at $>200^{\circ}$ in $0.08 \mathrm{mJy} /$ Beam $(\sim 3 \sigma)$. We discuss asymmetric structures in Section 3.4 in more detail by directly analyzing the data in visibility domain.

In the rest of this section, we show the difference between the upper $(233.0 \mathrm{GHz})$ and lower $(216.7 \mathrm{GHz})$ band data. In Figure 4, we compare the real parts of the visibility of upper and lower band data. The visibility data are deprojected using the inclination and position angle derived earlier. Then, the data with similar $u v$-distance are binned and averaged. The bin width are $10 k \lambda$ for $\rho<1200 k \lambda$ while $20 k \lambda$ for $\rho>1200 k \lambda$, where $\rho$ is the $u v$-distance of the deprojected visibility data. The error bars in the figure indicate the standard deviation of the data divided by the square root of the number of data. The amplitudes of the visibility are clearly different at small $u v$-distances, namely $\rho<200 k \lambda$, which corresponds to a spacial scale of $\sim 1$ arcsec. Moreover, one can find that the visibility of the upper band data is larger than that of the lower band data around the peak around $\rho=700 k \lambda$. The details of statistics of the data are described in Appendix A.
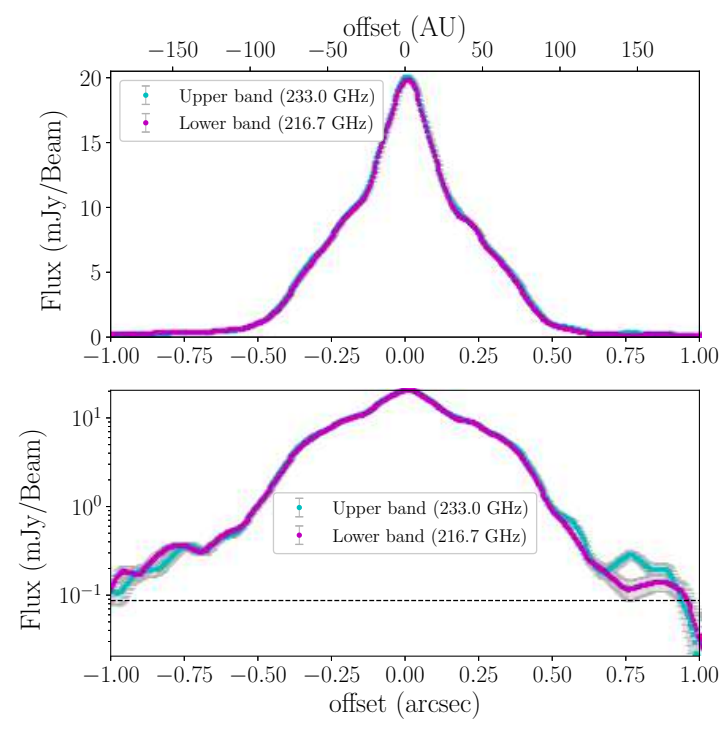

Figure 5. Intensity distributions along the major axis for the upper and lower band data. The error bar denotes $1 \sigma$ noise level $(=0.029 \mathrm{mJy} / \mathrm{Beam})$ and the dashed horizontal line denotes $3 \sigma$ noise level (0.087 mJy/Beam).

Figure 5 compares the intensity distributions along the major axis for the upper and lower band data. The intensity of the upper band data is slightly larger than that of the lower band data. Indeed, for the upper band data, the total flux density with $>3 \sigma(0.087 \mathrm{mJy})$ emission is $516.16 \mathrm{mJy}$ and for the lower band data, it is $430.20 \mathrm{mJy}$. We note that at the offset $\simeq 0.75$ arcsec, the profile is different in the upper and lower band data, which can be related to the asymmetric structures discussed in Section 3.4.

\section{MCMC MODELING OF DUST CONTINUUM EMISSION}

\subsection{Model description}

To examine the structure of the disk in detail, we performed fitting for dust continuum emission in the visibility domain with a simple disk model. As described in the previous section, the disk could have two peaks at $\sim 0.3$ arcsec and 0.75 arcsec. Moreover, we also include an unresolved small cavity. By motivated from the observed features, we adopted a simple power-law intensity profile with exponential cutoff with two Gaussian bumps, two intensity enhanced/depleted regions and an inner cavity, as described below:

$$
\begin{aligned}
I(R) \propto\left[f_{I}\left(\frac{R}{R_{\mathrm{c}}}\right)^{-\gamma} \exp \left[-\left(\frac{R}{R_{\mathrm{c}}}\right)^{\zeta}\right]\right. \\
\left.+\sum_{i=1}^{2} H_{i} \exp \left[-\left(\frac{R-R_{\mathrm{rc}, \mathrm{i}}}{W_{i}}\right)^{2}\right]\right]
\end{aligned}
$$


where

$$
f_{I}= \begin{cases}\delta_{\mathrm{cav}} & 0<R<R_{\mathrm{cav}} \\ 1 & R_{\mathrm{cav}}<R<R_{\mathrm{g}, \text { in }, 1} \\ \delta_{\mathrm{gc}, 1} & \left.R_{\mathrm{g}, \text { in }, 1}<R<R_{\mathrm{g}, \text { out }, 1}\right) \\ \delta_{\mathrm{gc}, 2} & \left.R>R_{\mathrm{g}, \text { out }, 1}\right)\end{cases}
$$

The total flux that the intensity given by Equation (2) is integrated over the entire disk is normalized to be $F_{\text {tot }}$ which is one of the parameters of the model. The intensity distribution of Equation (2) has 16 parameters, namely, two exponents $\gamma$ $\zeta$, the depth and radius of the inner cavity $\delta_{\text {cav }}, R_{\text {cav }}$, characteristic radius $R_{\mathrm{c}}$, total intensity of the disk $F_{\text {tot }}$, and parameters of substructures: $R_{\mathrm{g}, \text { in }, 1}, R_{\mathrm{g}, \text { out }, 1}, \delta_{\mathrm{gc}, 1}, \delta_{\mathrm{gc}, 2}$ for two enhanced/depleted regions and $R_{\mathrm{rc}, 1}, W_{1}, H_{1}, R_{\mathrm{rc}, 2}, W_{2}, H_{2}$ for two Gaussian bumps.

\subsection{Fitting approach}

We fit the observation data with the model of Equation (2) in the visibility domain. In this modeling, we focus on a symmetric structure, following Zhang et al. (2015). In the following, $\rho$ indicates the deprojected baseline in the visibility domain. The likelihood function is defined by

$$
\chi^{2}=\sum_{k}^{N}\left(\frac{\operatorname{Re}(\bar{V})_{\mathrm{obs}, \mathrm{k}}-\operatorname{Re}(\bar{V})_{\text {model }, \mathrm{k}}}{\sigma_{\mathrm{obs}, \mathrm{k}}}\right)^{2}
$$

where $k$ indicates the index of the radial bin and $N$ is the total number of radial bins. We take an average within the bin in radial and azimuthal direction in visibility domain (the overline indicates the average). The bin size is $10 k \lambda$ for $\rho>1200 k \lambda$, and $20 k \lambda$ for $\rho<1200 k \lambda$. Since the amplitude of the visibility is comparable with the noise level in $\rho>2000 k \lambda$, we used the visibility in the range of $\rho<2000 k \lambda$ in this modeling. The real part of the visibility is denoted by $\operatorname{Re}(V)$ and the subscript 'model' and 'obs' indicate the quantities of model and observation, respectively. The standard deviation of the averaged real part of the visibility $\sigma_{\mathrm{obs}, \mathrm{i}}$ is calculated by dividing the standard deviation of azimuthal direction in the visibility domain by the square root of the number of data within the bin.

For the fitting, we utilized the public python code vis_sample (Loomis et al. 2017). We used the Markov Chain Monte Carlo (MCMC) method in the emcee package (Foreman-Mackey et al. 2013). We carried out the fitting with the MCMC method with $\chi^{2}$ given by Equations (4). In the MCMC fitting, we run 1000 steps with 100 walkers after the burnin phase with 1000 steps (2000 steps in total).

\section{3. fitting result}

We found that the C43-4 (compact configuration) data is slightly scattered as compared with the C43-8 (sparse configuration) data, and the data is slightly statistically different

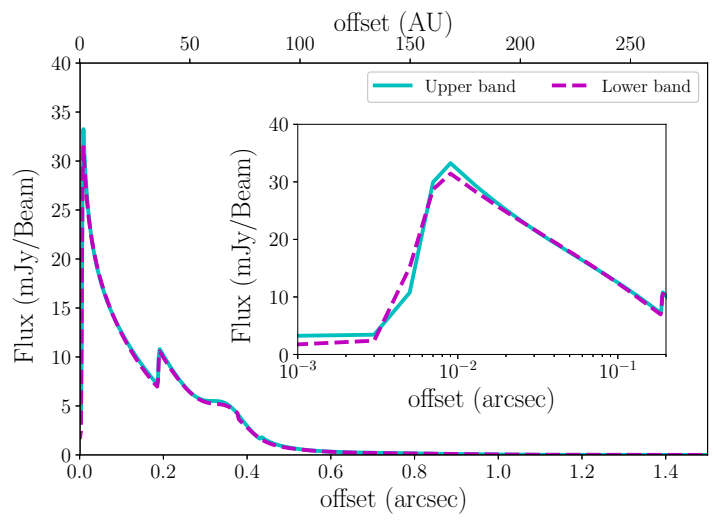

Figure 6. The shapes of the best-fit model given by parameters listed in Table 2, for the upper and lower band data. The inset is the zoom in of the region of offset $<0.2$ arcsec.

around $\rho=200 k \lambda$ (see Appendix A). Because of this difference between the C43-4 and C43-8 data, the reduced $\chi^{2}$ of the best fit model is much deviated from unity, when the data are combined. If only C43-4 data is used, the resolution is not enough to identify substructures. Hence, we used only the $\mathrm{C} 43-8$ data for the MCMC fitting ${ }^{2}$. We performed the MCMC fitting for the upper and lower band data separately.

The total flux density of the images synthesized by only the C43-8 data is $10 \%-20 \%$ smaller than that by both $\mathrm{C} 43-$ 8 and C43-4 data due to missing flux. However, since the visibilities of the $\mathrm{C} 43-8$ data are quite similar to these that combined by the C43-8 and C43-4 data, as shown in Figure 19), excluding the C43-4 data could not affect the fitting results.

The fitting results are summarized in Table 2. The best fit parameters for the upper and lower band data are slightly different, especially on the total flux density, and the parameters related the outer structure, namely, $\zeta, \delta_{g, 2}$ and the parameters of the outer peak $\left(R_{\mathrm{rc}, 2}, W_{2}, H_{2}\right)$, and the depth of the inner cavity $\left(\delta_{\text {cav }}\right)$.

Figure 6 shows the best-fit models for the upper and lower band data. They look very similar, as both have small cavity with radius $\sim 1$ au (corresponding to $R_{\text {cav }}$ ), a bump-like excess emission at $R \sim 0.2-0.4$ arcsec with double peaks at $0.2 \operatorname{arcsec}$ (corresponding to $R_{\mathrm{g}, \text { in }, 1}, \simeq 40 \mathrm{au}$ ) and 0.35 arcsec (corresponding to $R_{\mathrm{rc}, 1}, \simeq 70 \mathrm{au}$ ). The fitting results of both upper and lower bands indicate that the there is a cavity with the radius of $\sim 0.005 \operatorname{arcsec}(\sim 1 \mathrm{au})$. Since the size of inner cavity is much smaller than the spatial resolution with $\rho<2000 k \lambda$, we consider that this structure should be confirmed in future observations. For the outer region, though

\footnotetext{
${ }^{2}$ When both the C43-4 and C43-8 data are used, the reduced $\chi^{2}$ is $\sim 6$, though the best fit parameters are similar to these shown in table 2. This large reduced $\chi^{2}$ is mainly due to points around $\rho=200 k \lambda$.
} 
Table 2. Fitting results for $\mathrm{C} 43-8$ data

\begin{tabular}{|c|c|c|c|c|c|c|}
\hline \multirow{3}{*}{ Parameters } & \multicolumn{3}{|c|}{ Upper band (233.0 GHz data) } & \multicolumn{3}{|c|}{ Lower band (216.7 GHz data) } \\
\hline & \multirow[t]{2}{*}{ Best fit } & \multicolumn{2}{|c|}{ Range } & \multirow[t]{2}{*}{ Best fit } & \multicolumn{2}{|c|}{ Range } \\
\hline & & Min & Max & & Min & Max \\
\hline$\gamma$ & $0.349_{+0.030}^{-0.025}$ & 0.0 & 0.5 & $0.280_{+0.023}^{-0.021}$ & 0.0 & 0.5 \\
\hline$\zeta$ & $1.946_{+0.079}^{-0.061}$ & 1.8 & 2.3 & $1.489_{+0.062}^{-0.041}$ & 1.0 & 2.0 \\
\hline$R_{\mathrm{c}}(\mathrm{au})$ & $51.160_{+2.249}^{-1.777}$ & 38.0 & 57.0 & $45.881_{+1.710}^{-1.499}$ & 26.6 & 76.0 \\
\hline$R_{\text {cav }}(\mathrm{au})$ & $1.296_{+0.348}^{-0.490}$ & 0.0 & 1.9 & $0.986_{+0.353}^{-0.450}$ & 0.0 & 1.9 \\
\hline$R_{\mathrm{g}, \mathrm{in}, 1}(\mathrm{au})$ & $35.908_{+0.216}^{-0.211}$ & 30.4 & 45.6 & $35.630_{+0.245}^{-0.314}$ & 30.4 & 45.6 \\
\hline$R_{\mathrm{g}, \text { out }, 1}(\mathrm{au})$ & $81.994_{+3.488}^{-2.973}$ & 57.0 & 114.0 & $71.894_{+1.804}^{-1.949}$ & 57.0 & 114.0 \\
\hline$R_{\mathrm{rc}, 1}(\mathrm{au})$ & $67.046_{+0.216}^{-0.240}$ & 57.0 & 76.0 & $68.310_{+0.775}^{-0.742}$ & 57.0 & 76.0 \\
\hline$W_{1}(\mathrm{au})$ & $10.835_{+0.613}^{-0.553}$ & 0.4 & 38.0 & $10.990_{+1.308}^{-0.621}$ & 0.4 & 38.0 \\
\hline $\ln \left(H_{1}\right)$ & $-0.627_{+0.026}^{-0.019}$ & -1.0 & 0.5 & $-0.826_{+0.036}^{-0.024}$ & -1.0 & 0.5 \\
\hline$R_{\mathrm{rc}, 2}(\mathrm{au})$ & $122.223_{+5.861}^{-6.281}$ & 95.0 & 152.0 & $146.630_{+4.100}^{-6.825}$ & 114.0 & 190.0 \\
\hline$W_{2}(\mathrm{au})$ & $56.530_{+3.597}^{-3.527}$ & 11.4 & 76.0 & $42.890_{+5.221}^{-3.547}$ & 11.4 & 76.0 \\
\hline $\ln \left(H_{2}\right)$ & $-1.708_{+0.051}^{-0.031}$ & -3.0 & -1.0 & $-2.036_{+0.060}^{-0.050}$ & -3.0 & -1.5 \\
\hline $\ln \left(\delta_{\mathrm{cav}}\right)$ & $-1.055_{+0.498}^{-0.763}$ & -3.0 & -0.0 & $-1.517_{+0.781}^{-0.845}$ & -3.0 & -0.0 \\
\hline $\ln \left(\delta_{\mathrm{g}, 1}\right)$ & $\begin{array}{c}0.194_{+0.009}^{-0.010} \\
\end{array}$ & -0.5 & 1.0 & $0.199_{+0.008}^{-0.010}$ & -0.5 & 1.0 \\
\hline $\ln \left(\delta_{\mathrm{g}, 2}\right)$ & $0.305_{+0.037}^{-0.045}$ & -1.0 & 1.0 & $0.091_{+0.055}^{-0.063}$ & -1.0 & 0.5 \\
\hline$F_{\text {tot }}(\mathrm{mJy})$ & $\begin{array}{r}493.995_{+0.527}^{-0.553} \\
\end{array}$ & 470.0 & 510.0 & $\begin{array}{r}418.431_{+0.558}^{-0.593} \\
\end{array}$ & 390.0 & 430.0 \\
\hline
\end{tabular}

NOTE- Error range of the best parameters are estimated by $\pm 1 \sigma$.

the parameters such as $\gamma, \zeta$, and $R_{\mathrm{rc}, 2}$ are slightly different, the profiles agree with each other.

Figure 7 compares the visibility of the model and observation. As can be seen in the figure, the models well reproduce the observed visibility, and the reduced $\chi^{2}$ for the model of the upper band data is 1.58 and that of the lower band data is 1.28 , respectively.

Figure 8 compares the model and the observation in the image. The model image is first converted to the ALMA measurement set by vis_simple with the observed measurement set and we made a mock observational image by the same procedure of the imaging of the observed data with the parameters listed in Table 1. In Figure 8, we show the intensity distributions along the major axis in the mock observational image (model) and the observed image. In the bottom panel of the figure, we show the residual between the model and the observational data. We calculated the residual as the subtraction between the model and the observation in the visibility domain, by using vis_sample. The visibility of the residual is converted by tclean task with the imaging parameters listed in Table 1 . Around the center, one can see the residual which is larger than $3 \sigma$, though the residual is smaller or comparable with the $3 \sigma$ level in other regions. This discrepancy is related to the asymmetry which is indicated by the difference between the structures along the major and minor axes shown in Figure 2.
Figure 9 shows the map of the residual between the model and the observation in the upper and lower band data. The pattern of the residual in the upper and lower band data are similar to each other. One can see significant residuals around the center, which is also shown by Figure 8 . Moreover, the residual map shows positive and negative structures at the upper left (R.A. offset $\simeq-0.4$ arcsec, Dec. offset $\simeq 0.2 \mathrm{arcsec})$. The amplitudes of those structures are larger than $5 \sigma$, which can indicate the real asymmetric structures.

\subsection{Asymmetric structure}

As shown in the previous subsection, the residual map between the model and the observation indicates the asymmetric structures at the center and the outer disk. Here we further investigate this asymmetry of the disk, by using a modelindependent analysis.

In the visibility domain, the visibility is expressed by

$$
V(\vec{\rho})=\iint I(\vec{R}) e^{-j \vec{R} \cdot \vec{\rho}} d \vec{R},
$$

where $j=\sqrt{-1}$ is the imaginary unit and $\vec{\rho}$ and $\vec{R}$ indicate the position vectors in the deprojected uv plane and the image, $I(\vec{R})$ is the intensity distribution. When $I(\vec{R})$ is axisymmetric, we can express the visibility as

$$
V(\rho)=2 \pi \int I(R) J_{0}(R \rho) R d R,
$$



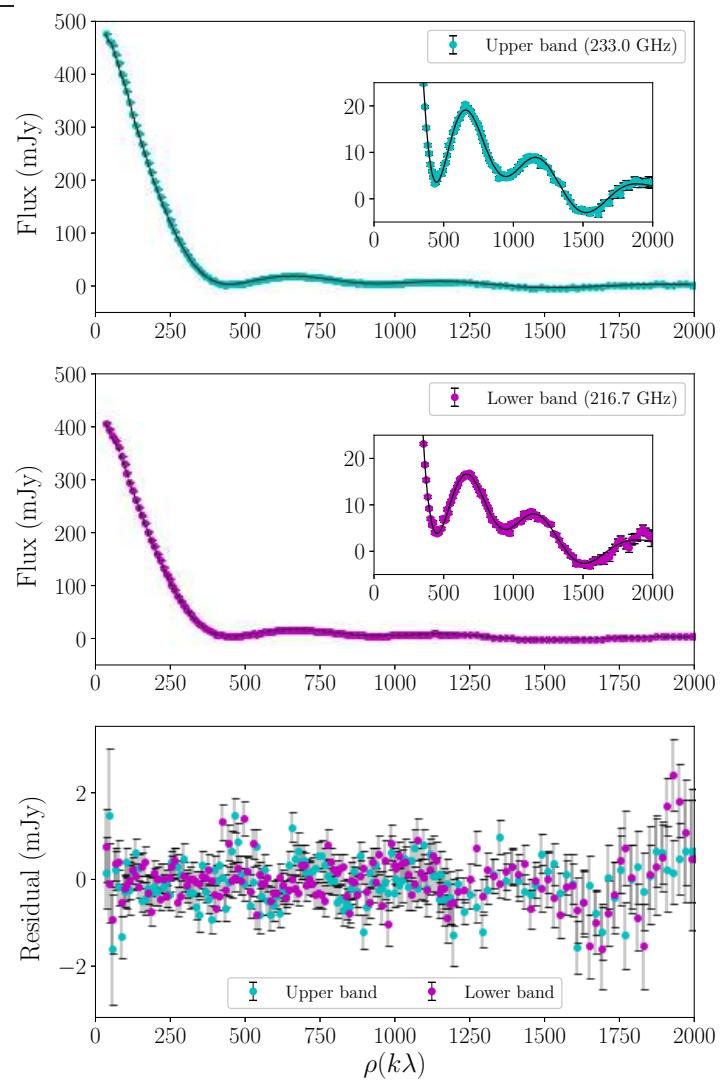

Figure 7. Real part of the visibilities for the observation (dot) and best-fit-model (solid)) for the upper and lower band data, in the upper and middle panels, respectively. The inset shows the zoom-up view of the region with $<30 \mathrm{mJy}$. In the lower panel, we show the different of the visibilities between the observation and the model. The error bars of the observational data and residuals are estimated by $1 \sigma$ deviation of the average.

where $J_{0}(k)$ is 0th-order Bessel function of the first kind. The visibility of the axisymmetric disk has only a real part. The image does not change if the disk is $180^{\circ}$ rotated against the disk center. In mathematics, a $180^{\circ}$ rotated image has the visibility which is the complex conjugate of that of the original image. Hence, the difference between the original and $180^{\circ}$ rotated images has only imaginary part, namely, twice the imaginary part of the original image. When the system does not have a significant asymmetric structure, the difference is almost zero because the imaginary part of the visibility of the original image is very small. On the other hand, when the disk has asymmetric structures, we could see some residual between the original and $180^{\circ}$ rotated images, which corresponds to the imaginary part of the visibility. This approach of investigating asymmetric structures is totally model-independent.

We produced the synthesized image using all the data, namely, the C43-4 and C43-8 data in upper and lower bands. Figure 10 shows the azimuthally averaged imaginary part of
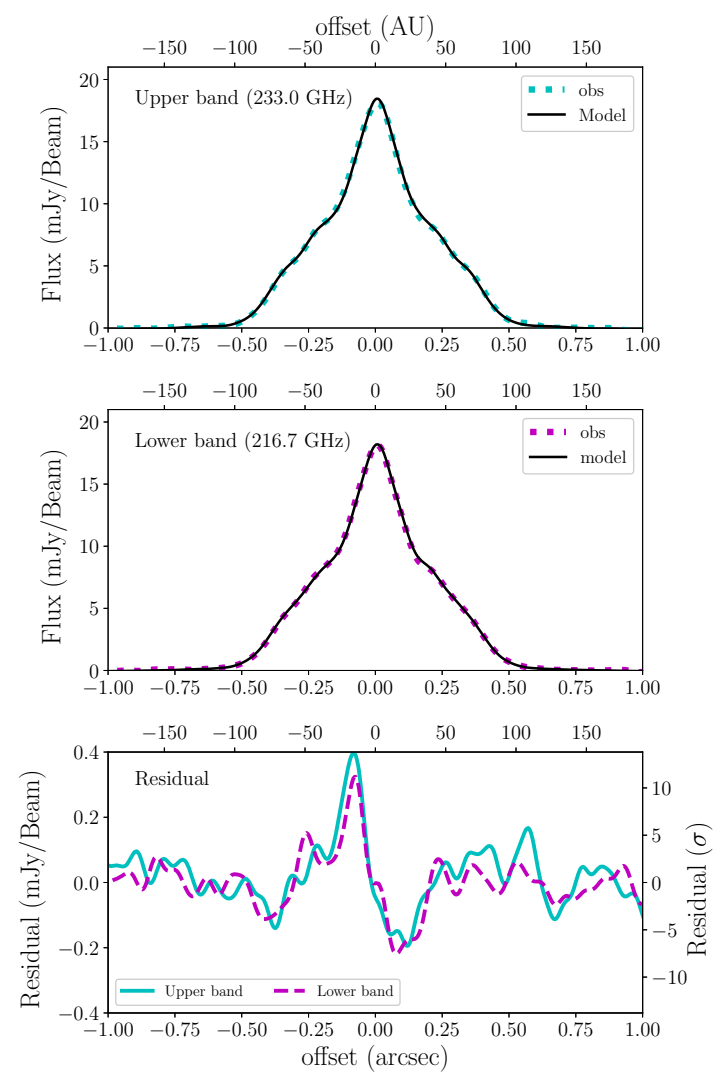

Figure 8. Intensity distributions along the major axis in the models and observations for the upper band data(upper) and the lower band data (middle). The bottom panel shows the residuals between the model and the observation, along the major axis.

the visibility. The average is calculated in the same way as in Figure 4. The imaginary part of the visibility is much fainter than the real part. However, one can see the significant signals as large as a few mJy, at $\rho \lesssim 300 k \lambda$, which implies the asymmetric structure with the scale of $\gtrsim 0.7$ arc$\sec (\gtrsim 140 \mathrm{au})$. Figure 11 shows the synthesized image produced only from the imaginary part of visibility data. We can find the asymmetric structure at the center and that at the upper left (R.A offset $\simeq-0.4 \operatorname{arcsec}(\simeq-80$ au) and Dec. offset $\simeq 0.2 \operatorname{arcsec}(\simeq 40 \mathrm{au}))$, which is consistent with the difference btween the observed image and the axisymmetric model shown in Figure 9. In addition, we see structures at the lower right, namely, R.A offset $\simeq 0.4$ arcsec and Dec. offset $\simeq-0.2$ arcsec. The Fourier transform of pure imaginary function must be an odd function, which means that there is a counter part in the opposite location in the synthesized image. If the asymmetric structure indicated by the imaginary part is real, we should find a signal at the same location with the model-subtracted image. Hence, we consider that the structure at the lower right could be the counter part of the structure at the upper left. 


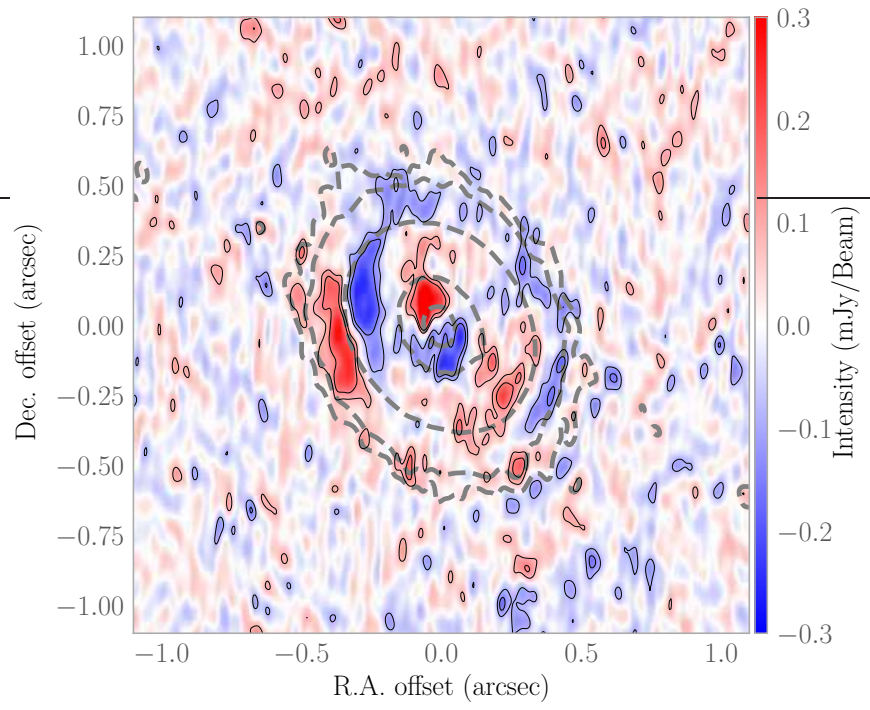

(a) Residual at $233.0 \mathrm{GHz}$

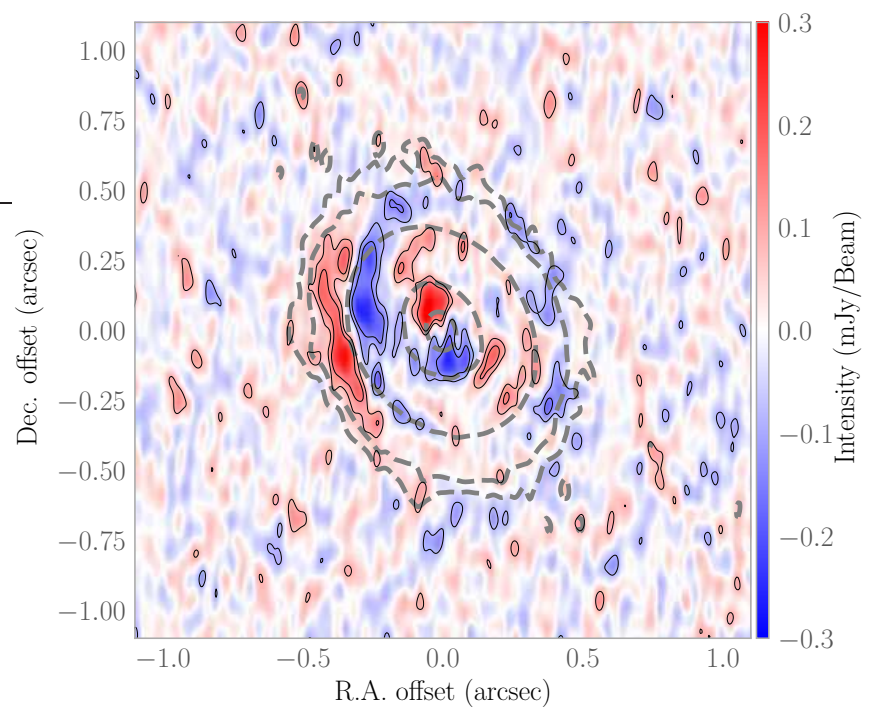

(b) Residual at $216.7 \mathrm{GHz}$

Figure 9. Residual between the observational data and model at $233.0 \mathrm{GHz}$ (a), and at $216.7 \mathrm{GHz}$ (b). The contour indicates the levels of $\pm 3 \sigma$ and $\pm 5 \sigma$. The thick dashed contour lines indicate the observed intensity distribution, which are $0.087 \mathrm{mJy} / \mathrm{Beam}(3 \sigma), 0.29 \mathrm{mJy} / \mathrm{Beam}(10 \sigma)$, $2.9 \mathrm{mJy} /$ Beam $(100 \sigma), 8.7 \mathrm{mJy} /$ Beam $(300 \sigma)$, and $14.5 \mathrm{mJy} /$ Beam $(500 \sigma)$ from the outside.

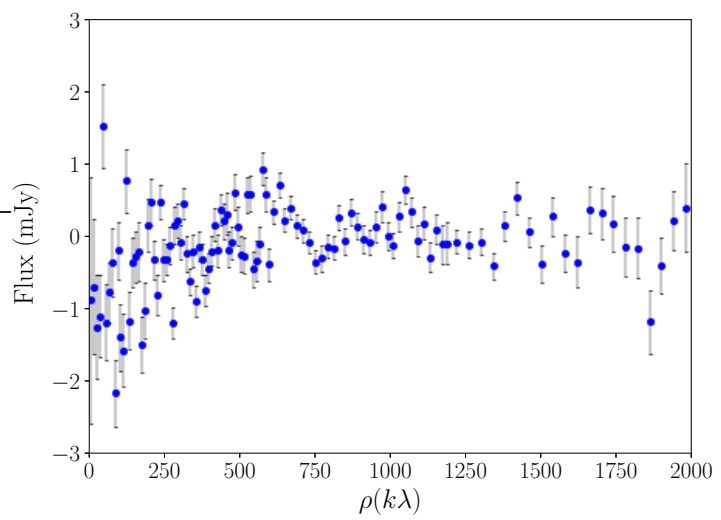

Figure 10. Imaginary part of the visibility combined from all data.

As described in Section 2 and in the result shown above, we determined the phase center by the Gaussian fitting with CASA tool uvmodelfit. However, the above analysis of the imaginary part of the visibility depends on the choice of the phase center. Figure 12 shows how the images (deprojected to the disk plane) produced from pure imaginary visibility change with the choice of the phase center. In the figure, we shift the center in \pm 2 mas ( $\pm 0.38 \mathrm{au}$ ) in horizontal and vertical directions from the phase center of Figure 11. The image is shifted in the visibility domain by the phase shift defined as $\exp [2 \pi(u d x+v d y)]$, where $u$ and $v$ are the spatial frequencies and $d x$ and $d y$ are shift values in R.A and Dec. directions, respectively. It is reasonable to assume that the disk structure is almost axisymmetric, despite the disk has some asymmetric structures. Under this assumption, the

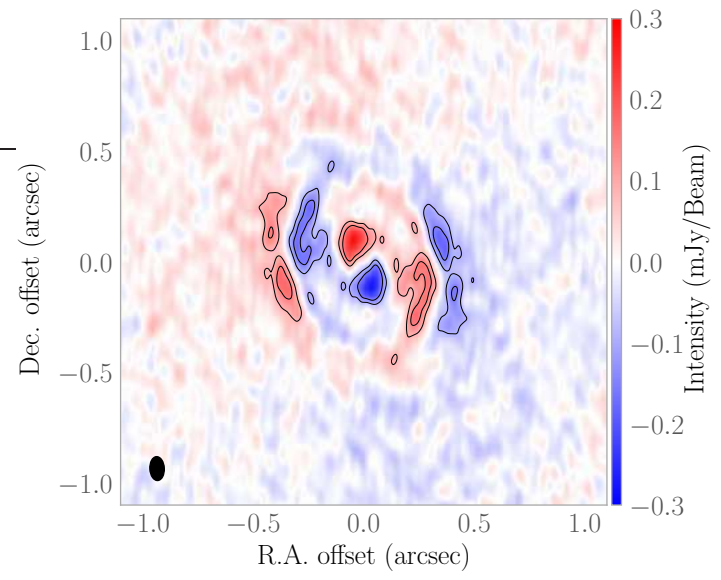

Figure 11. Image synthesized from the imaginary part of the visibility shown in Figure 10. The contour indicates the levels of $\pm 3 \sigma$ $( \pm 0.087 \mathrm{mJy} /$ Beam $)$ and $\pm 5 \sigma( \pm 0.145 \mathrm{mJy} /$ Beam $)$.

image deprojected from the imaginary part with the 'correct' phase center has the minimum root mean square of the intensity. We calculated the sum of the root mean square value of the intensity at each pixel within the radius of 0.6 arcsec (114 au) from the center, which is labeled at the upper left corner at each panel (labeled by RMS). The figure with our fiducial phase center has the minimum value of RMS among the listed panels. Hence, the phase center determined by the Gaussian fit is consistent with the center which minimalize the asymmetry. On the other hand, in the panel at the upper left corner ( $\mathrm{dx}=2$ mas and $\mathrm{dy}=2 \mathrm{mas})$, the asymmetric structure around the center is almost vanished. This indicates that 

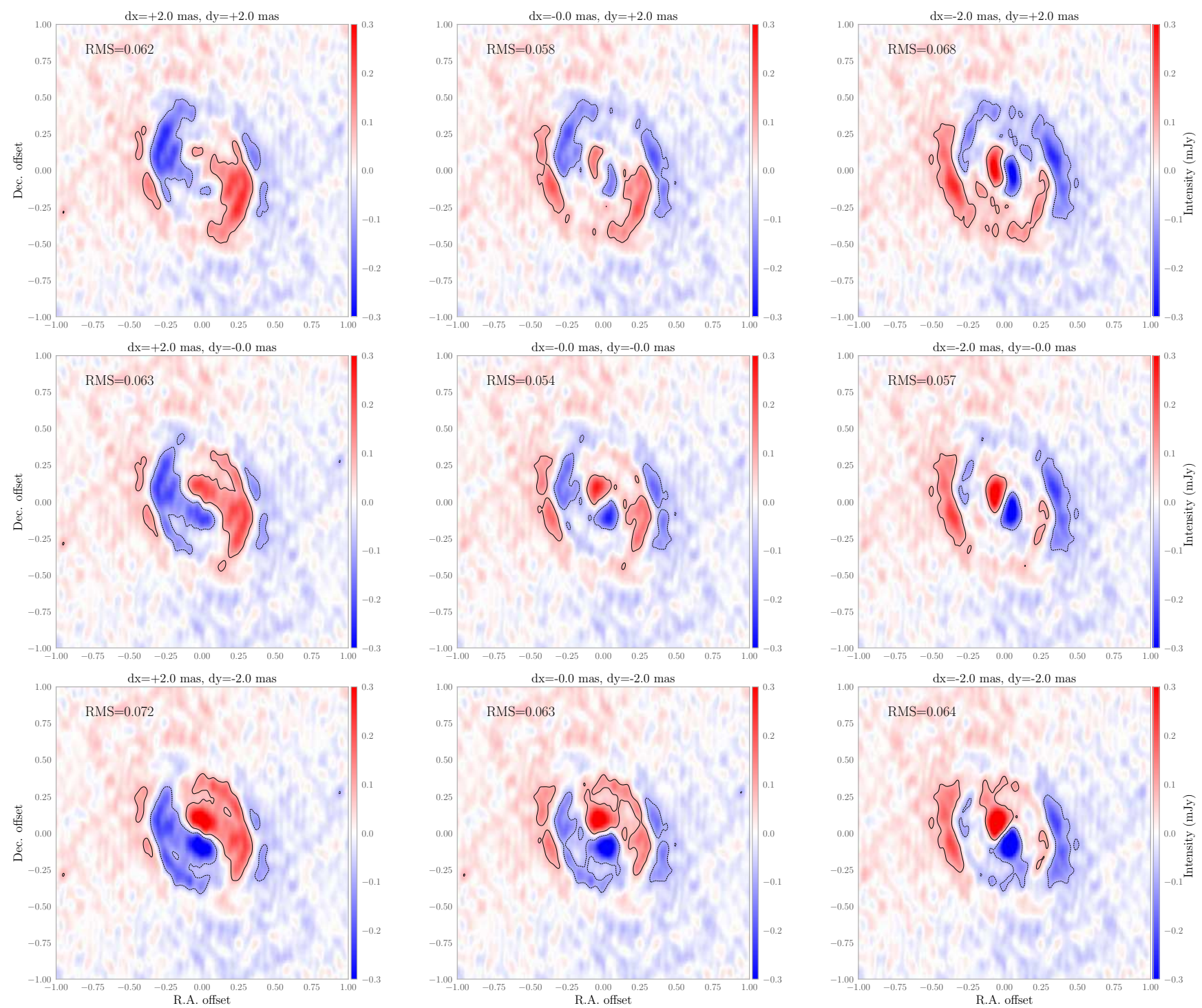

Figure 12. Images deprojected only from the imaginary part of the visibility as shown in Figure 11, but with the different phase center. The central figure is the same as that shown in Figure 11 with the same phase center. The horizontal coordinate of phase center is shifted in 2 mas ( -2 mas) from that of the central figure, in left (right) column, and the vertical coordinate of the phase center is shifted in 2 mas $(-2$ mas) in the top (bottom) row. In the middle column (row), the horizontal (vertical) coordinate of the phase center is the same as that of the center figure.

the center of the inner structure is shifted to the center of the outer structure.

\section{MODELING BY RADIATIVE TRANSFER SIMULATIONS}

\subsection{Setup and model description}

We now have a model for the intensity distribution of the disk around WW Cha. In this section, we discuss the physical condition (e.g., dust surface density, size distribution of the dust grains, and temperature) of the disk, by using radiative transfer simulations with RADMC-3D ${ }^{3}$ (Dullemond et al. 2012). We setup a model of an axisymmetric dust surface density distribution which is motivated by the intensity distribution derived in the previous section as

$$
\begin{aligned}
\Sigma(R)=\Sigma_{0} & {\left[f_{I}\left(\frac{R}{R_{\mathrm{c}}}\right)^{-s} \exp \left[-\left(\frac{R}{R_{\mathrm{c}}}\right)^{\zeta}\right]\right.} \\
+ & \left.\sum_{i=1}^{2} H_{i} \exp \left[-\left(\frac{R-R_{\mathrm{rc}, \mathrm{i}}}{W_{i}}\right)^{2}\right]\right]
\end{aligned}
$$

\footnotetext{
${ }^{3} \mathrm{http}: / /$ www.ita.uniheidelberg.de/ dullemond/software/radmc-3d/index.html
} 
Here $f_{I}$ is defined by Equation (3), $\zeta, R_{\mathrm{c}}, H_{i}, W_{i}, R_{\mathrm{rc}, \mathrm{i}}$, and parameters in $f_{I}\left(\delta_{\mathrm{cav}}, \delta_{\mathrm{gc}, 1}, \delta_{\mathrm{gc}, 2}, R_{\mathrm{cav}}, R_{\mathrm{g}, \text { in }, 1}, R_{\mathrm{g}, \text { out }, 1}\right)$ are fixed to the values given in Table 2 (for C43-8 data). For the parameter $s$ that is related to the slope of the dust surface density, we use $s=0$, which is different from the bestfit parameter of $\gamma$ which is $\sim 0.35$. We confirm that the choice of $s$ hardly affects the estimates of physical parameters. When we use $s=0.5$, the mid-plane temperature is affected only by a few Kelvin. The parameter $\Sigma_{0}$ controls the total mass of the dust $M_{\text {dust }}$ and when $\Sigma_{0}=1.5 \mathrm{~g} / \mathrm{cm}^{2}$, $M_{\text {dust }}=3 \times 10^{-3} M_{\odot}$.

We first vary the stellar luminosity and $\Sigma_{0}$ and check the agreement with observations in order to address the uncertainty of the estimate of the disk physical parameters. We then vary the dust size in order to address the spectral index distribution. Here we present a physical disk and star model that reasonably matches observations. Full modeling studies that derive the uncertainties of all the parameters are beyond the scope of this paper.

Rest of the setup of the simulation is as follows. In the vertical direction, we adopt a Gaussian shape distribution, namely,

$$
\rho(R, z)=\frac{\Sigma(R)}{\sqrt{2 \pi} h} \exp \left(-\frac{z^{2}}{2 h^{2}}\right),
$$

where $h$ is the scale height of the dust layer. The dust scale height can be smaller than that of gas structure, due to dust settling (e.g., Nakagawa et al. 1986). Hence, we consider two dust components:one is a small dust component with size distribution $\propto s^{-3.5}$, where $s$ is the size of the grains, and the minimum and maximum sizes are $0.1 \mu \mathrm{m}$ and $0.1 \mathrm{~mm}$, respectively. The other is a large dust component with its size having Gaussian distribution in logarithmic space. The peak of the size distribution $s_{\text {d, large }}$ is $1 \mathrm{~mm}$ with the smallest size $0.6 \mathrm{~mm}$ and with the largest size of $1.7 \mathrm{~mm}$. We assume that the scale height of the large grains is 0.1 times the scale height of the gas. The mass ratio between the small and large grains is set to be 1:9. We adopt the same compositions of the dust grains to that adopted in Birnstiel et al. $(2018)^{4}$. The absorption and scattering coefficients for each component are averaged by the size distribution.

The mass and the surface temperature of the central star are set to $1.2 M_{\odot}$ and $4350 \mathrm{~K}$, respectively (Luhman 2007). By the SED modeling, the stellar luminosity is estimated by $11 L_{\odot}$ (Garufi et al. 2020). Since WW Cha is a young newborn star, however, it could be still embedded in the core (Ribas et al. 2013; Garufi et al. 2020). In such a case, the luminosity may be underestimated due to high extinction (Follette et al. 2015). Hence, we also consider the case with $L_{*}=22 L_{\odot}$.

\footnotetext{
${ }^{4}$ The optical constant file was provided by Dr. Ryo Tazaki.
}

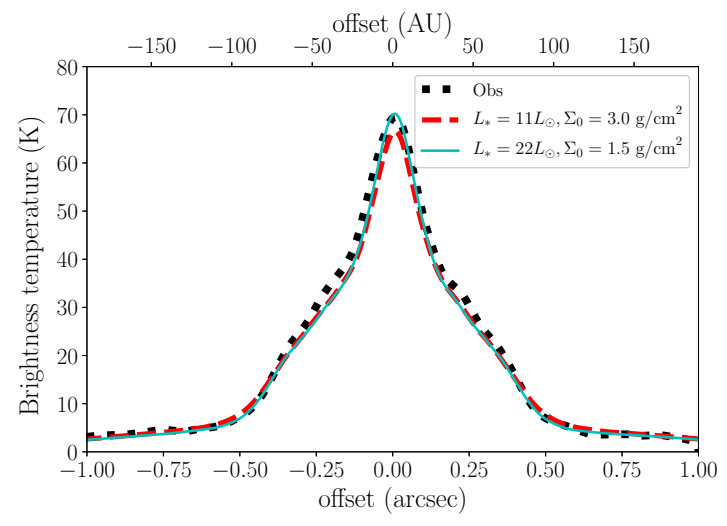

Figure 13. Brightness temperature distributions along the major axis of the disk, for the observation (black dot), the results of the simulations with $L_{*}=11 L_{\odot}, \Sigma_{0}=3 \mathrm{~g} / \mathrm{cm}^{2}$ (red dashed) and with $L_{*}=22 L_{\odot}, \Sigma_{0}=1.5 \mathrm{~g} / \mathrm{cm}^{2}$ (cyan solid).

The radial coordinate extends from 0.1 au to 1000 au, which is divided into 256 meshes with logarithmic spacing. The azimuthal angle $\theta$ and polar angle $\phi$ are divided into 256 meshes in $0<\theta<2 \pi$ and in $0<\phi<\pi$ (the midplane is located at $\phi=\pi / 2$ ), respectively. We adopted $3 \times 10^{8}$ photons for thermal Monte Carlo radiative transfer and imaging, and for the SED, $10^{7}$ photons are adopted.

We first carried out radiative transfer simulations with the disk scale height calculated by the empirical formula given by Dong et al. (2018a), namely, $T=$ $220\left(L_{*} / 11 L_{\odot}\right)^{1 / 4}(R / 1 \mathrm{au})^{-0.5}$ (it is quite similar to Equation 1). After the first run, we calculate the temperature on the midplane at each radius and we preformed simulation again with the scale height given by the midplane temperature. We repeated the above cycles until the temperature distribution is converged. In our case, the temperature distribution is converged after 2-3 iterations.

For the imaging, we converted the output of RADMC-3D to the ALMA measurement set with observed measurement set by use of vis_sample. Then, we deprojected the image from the model measurement set with the imaging parameter listed in Table 1.

\subsection{Results}

\subsubsection{Intensity and spectral energy distribution}

Figure 13 compares the brightness temperatures given by the observations and simulations. With $L_{*}=11 L_{\odot}$, we need $\Sigma_{0}=3 \mathrm{~g} / \mathrm{cm}^{2}$ which corresponds to the total dust mass of $7 \times 10^{-3} M_{\odot}$. In this case, the disk is highly gravitationally unstable in most part if the gas-to-dust mass ratio is 100 , as shown below. If the stellar luminosity is larger by a factor of two $\left(L_{*}=22 L_{\odot}\right)$, we found that $\Sigma_{0}=1.5 \mathrm{~g} / \mathrm{cm}^{2}$ is enough to reproduce the observation.

Figure 14 illustrates the SED at $0.3 \mu \mathrm{m}-2 \mathrm{~cm}$, given by the previous observations (Lommen et al. 2007, 2009; 


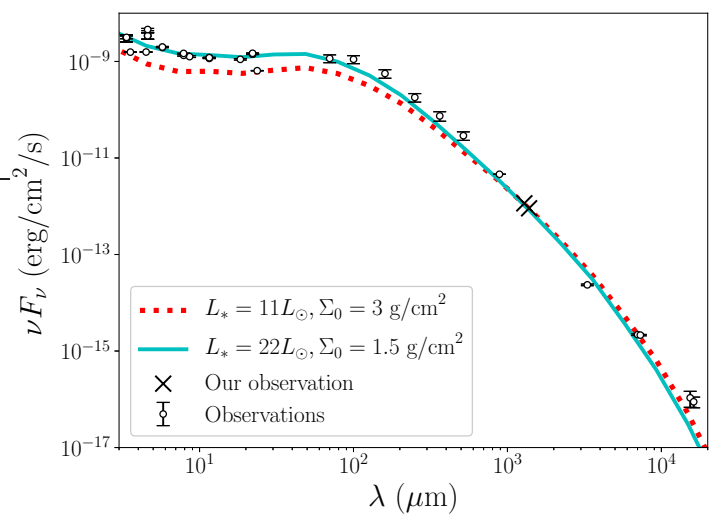

Figure 14. Spectral energy distribution given by observations (dots) and the simulations with $L_{*}=11 L_{\odot}, \Sigma_{0}=3 \mathrm{~g} / \mathrm{cm}^{2}$ (red dashed) and with $L_{*}=22 L_{\odot}, \Sigma_{0}=1.5 \mathrm{~g} / \mathrm{cm}^{2}$ (cyan solid). The crosses indicate the total fluxes given by our observation at $233 \mathrm{GHz}$ (494 mJy) and $216.7 \mathrm{GHz}$ (418 mJy). The observational data with $\lambda<1 \mathrm{~mm}$ are extracted from VizieR database (https://vizier.u-strasbg.fr/) and the references are in the main text.

Gutermuth et al. 2009; Ishihara et al. 2010; Cutri \& et al. 2014; Pascucci et al. 2016; Ribas et al. 2017), including our result, and the simulations. Both simulations with $L_{*}=$ $11 L_{\odot}$ and $22 L_{\odot}$ can reproduce the ALMA band 6 flux $(\sim 230 \mathrm{GHz})$. For fluxes at the longer wavelengths, namely $3 \mathrm{~mm}<\lambda<1 \mathrm{~cm}$, simulations agree with the observation. The fluxes of the simulations at $\lambda>1 \mathrm{~cm}$ is smaller than the observed flux, though it could be due to the contribution from the free-free emission from the star (Rodmann et al. 2006). In the case with $L_{*}=11 L_{\odot}$, the flux at far infrared wavelengths is about a factor of two smaller than the observed values. In this case, we need some contribution from the envelope to account for infrared flux. On the other hand, the case with $L_{*}=22 L_{\odot}$ can reproduce the fluxes from the infrared to the radio, by only the emission from the star.

\subsubsection{Dust density and temperature}

The stellar luminosity affects estimate on the mass of the gas disk, but it does not significantly affect the midplane temperature. In the upper panel of Figure 15, when $\Sigma_{0}=1.5 \mathrm{~g} / \mathrm{cm}^{2}$, we show the distribution of the dust surface density and Toomre's Q-value (Toomre 1964), assuming the gas-to-dust ratio of 100. The lower panel of Figure 15 shows the midplane temperatures given by simulations. The midplane temperature with $L_{*}=22 L_{\odot}$ is just about 1.16 times higher than that with $L_{*}=11 L_{\odot}$, roughly corresponding to $L_{*}^{1 / 4}$ dependence as expected from Equation (1).

We find that the disk is expected to have relatively low values of Toomre's Q-value. As shown in the upper panel of Figure 15, in the case of $\Sigma_{0}=1.5 \mathrm{~g} / \mathrm{cm}^{2}$, the Q-value is smaller than or close to unity at 20 au $<R<100$ au. In particular, the dust bump ( $40 \mathrm{au}-70 \mathrm{au}$ ) is gravitationally unstable be-
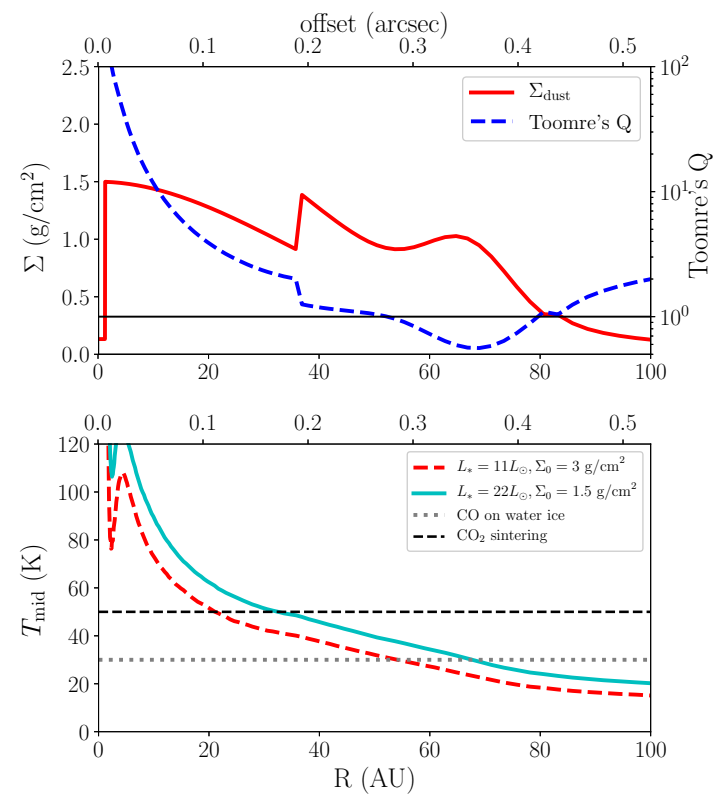

Figure 15. (Upper) Dust surface density and Toormre's Q-value (with gas-to-dust ratio being 100) in the case of $\Sigma_{0}=1.5 \mathrm{~g} / \mathrm{cm}^{2}$ (in the case of $\Sigma_{0}=3 \mathrm{~g} / \mathrm{cm}^{2}, \Sigma$ simply becomes two times larger and Q-value decreases with the increase of $\Sigma$.). The horizontal thin line indicates $Q=1$. (Lower) Midplane temperatures given by the radiative transfer simulations. The two horizontal lines indicate $50 \mathrm{~K}$ and $30 \mathrm{~K}$ from the top.

cause $Q \lesssim 1$ and the dust bump may fragment. In the case of $\Sigma_{0}=3 \mathrm{~g} / \mathrm{cm}^{2}$, the Q-value are decreased by a factor of two. In this case, the disk is still nearly gravitationally unstable and the fragmentation is expected at $R>10$ au and turbulence due to gravitational instability is also expected. However, no fragment-like structure is seen in the dust-continuum image. This may indicate that the gas-to-dust ratio is smaller than 100 in the region with $>20$ au. It is discussed in Section 5.1 .

We note that the temperatures at the peak locations are $~$ $30-50 \mathrm{~K}$, which is close to the freezeout temperature of the $\mathrm{CO}$ on water ice. This may be related to the origin of the bump structure, as discussed in Section 5.1.

\subsubsection{Spectral index}

Using the images given by the simulations at upper and lower bands, we produced the map of the spectral index by the use of tclean task with nterm=2. Although it is calculated from the very narrow frequency range, we may constrain the dust size distribution from it. The spectral index depends on the peak size of the large grain $s_{\mathrm{d} \text {,large }}$, rather than stellar luminosity and the dust surface density. Hence, we carried out additional simulations with $s_{\mathrm{d}, \text { large }}=0.5 \mathrm{~mm}$. In Figure 16, we show the spectral index map in the case of $s_{\mathrm{d} \text {,large }}=1.0 \mathrm{~mm}$ and $0.5 \mathrm{~mm}$, when $L_{*}=22 L_{\odot}$ and $\Sigma_{0}=1.5 \mathrm{~g} / \mathrm{cm}^{2}$. In the case of $s_{\mathrm{d}, \text { large }}=1.0 \mathrm{~mm}$, the spec- 

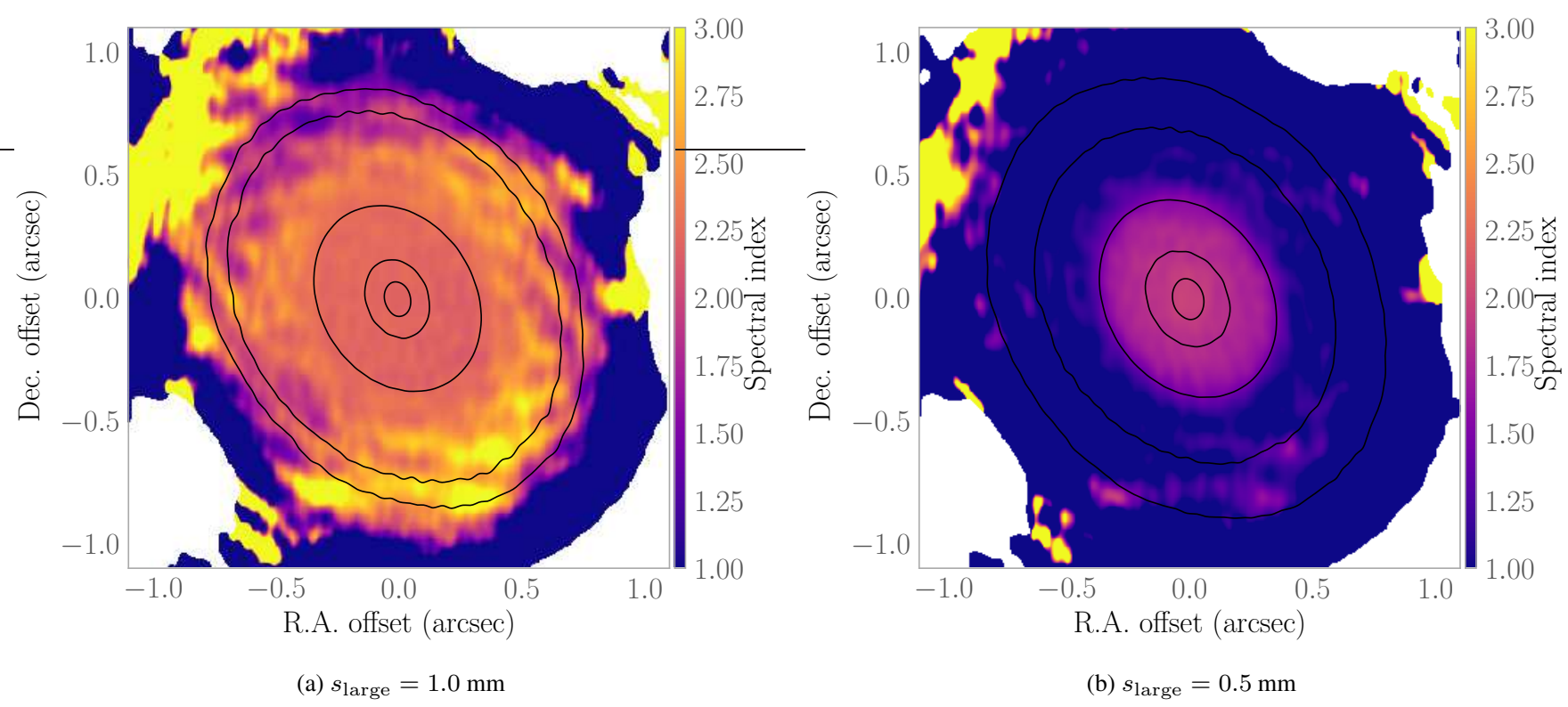

Figure 16. Spectral index maps given by radiative transfer simulations, with $s_{\mathrm{d}, \text { large }}=1 \mathrm{~mm}$ (left panel) and $s_{\mathrm{d}, \text { large }}=0.5 \mathrm{~mm}$ (right panel). The contours indicates the same flux levels shown in the panel (b) of Figure 1.

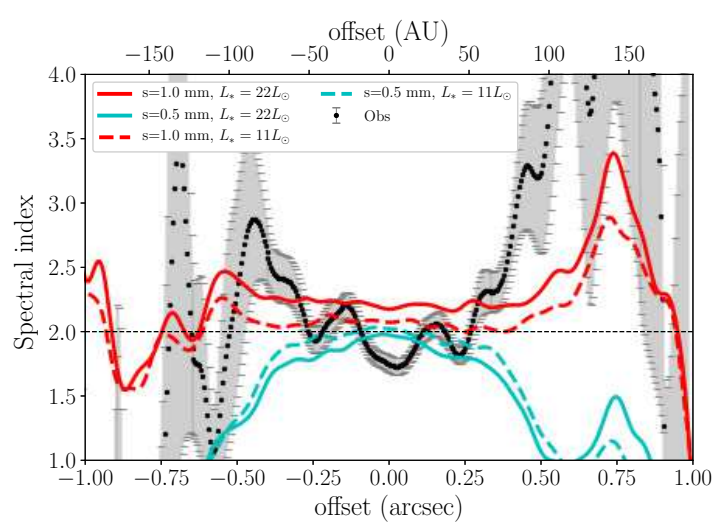

Figure 17. Distributions of the spectral index along the major axis in the case of $s_{\mathrm{d} \text {,large }}=1.0 \mathrm{~mm}$ (red) and $0.5 \mathrm{~mm}$ (cyan). In the upper panel, $L_{*}=22 L_{\odot}$ and $\Sigma_{0}=1.5 \mathrm{~g} / \mathrm{cm}^{2}$, while in the lower panel, $L_{*}=11 L_{\odot}$ and $\Sigma_{0}=3.0 \mathrm{~g} / \mathrm{cm}^{2}$. The dots indicates the observed one shown in the panel (d) of Figure 1.

tral index increases in the outer region, which agrees with the observations (panel (b) of Figure 1), but it is larger than 2 around the center. In the case of $s_{\mathrm{d} \text {,large }}=0.5 \mathrm{~mm}$, on the other hand, the spectral index is below 2 around the center, whereas it is much smaller than the observed index at the outer region.

Figure 17 compares the distributions of the spectral index along the major axis between the simulations and the observation. As can be seen in the figure, the distributions are similar between the case with $L_{*}=22 L_{\odot}$ and $\Sigma_{0}=1.5 \mathrm{~g} / \mathrm{cm}^{2}$ and the case with $L_{*}=11 L_{\odot}$ and $\Sigma_{0}=3.0 \mathrm{~g} / \mathrm{cm}^{2}$. In both the cases, for the outer region of offset $>0.25$ arcsec, the observational feature that the spectral index increases in the outer region is consistent with the radiative transfer calculations when $s_{\mathrm{d} \text {,large }}=1.0 \mathrm{~mm}$. For the inner region of $<0.25$ arcsec, the spectral index below 2 is consistent with the case of $s_{\mathrm{d} \text {,large }}=0.5 \mathrm{~mm}$.

The spectral index also depends on the size distribution of the large grains, though the intensity does not significantly depends on that. In Appendix $\mathrm{C}$, we demonstrate radiative transfer simulations with the large grains which have a power-law size distribution like that of the small grains. With the power-law distribution, the spectral index does not become below 2, even if the maximum size of the dust grains is $0.5 \mathrm{~mm}$. Hence, the log-normal size distribution of large grains may be preferred for the inner region. On the other hand, the power-law distribution may be preferred for the outer region as can be seen in Figure 23.

We should note that the spectral index discussed in this paper is provided from the very narrow range of the observed frequency within band 6 . The spatial variation of spectral index should be investigated by future observations at multiple wavelengths. In Appendix D, we show a few examples of the spectral index based on ALMA bands, which are calculated from the results of radiative transfer simulations. The spectral index can be different by the choice of the bands which are taken to calculate the spectra index. We may be able to constrain the dust size distribution from the spectral indexes in multiple bands.

\section{DISCUSSION}

\subsection{Origin of substructures}

\subsubsection{Ring}


We found the bump with double peaks at $40-80$ au from the central star by the model fitting in the visibility domain. One of the peaks is at $\sim 40$ au and the other is at $\sim 70 \mathrm{au}$.

The bump structure can be formed by dust trapping due to the pressure bump (e.g., Pinilla et al. 2012; Dullemond et al. 2018). However, the bump that is formed by the above mechanisms likely has a single peak, while the bump of our model has double peaks. If there are two pressure bumps, it might explain a shape with double peaks. Another possible location where a dust bump is likely to form is the outer edge of the planet-induced gap (e.g., Paardekooper \& Mellema 2004; Muto \& Inutsuka 2009; Zhu et al. 2012; Pinilla et al. 2015; Dong et al. 2015; Kanagawa et al. 2018). However, we did not detect any gap structure interior to the bump structure. Hence, it might not be the structure induced by the dust trap of the pressure bump and planetary gap.

One plausible scenario of producing a bump with double peaks uses the snowline. The bump can be formed due to volatile freeze-out altering the coagulation and fragmentation of dust grains (e.g., Zhang et al. 2015; Okuzumi et al. 2016). As shown in Figure 15, the temperature around the outer peak is about $30 \mathrm{~K}$, which is close to the freezeout temperature of CO at water ice Huang et al. (2018). Around the inner peak, the temperature is about $50 \mathrm{~K}$. Although it is not close to the condensation temperatures of major volatiles (e.g., $\mathrm{CO}, \mathrm{CO}_{2}$ ), it is close to the condensation temperature of $\mathrm{H}_{2} \mathrm{~S}$ (Zhang et al. 2015). Moreover, in $T \sim 50 \mathrm{~K}$, the sintering can occur for some species such as $\mathrm{H}_{2} \mathrm{~S}$ and $\mathrm{C}_{2} \mathrm{H}_{8}$ (Okuzumi et al. 2016). Hence, this bump with double peaks may be formed by the snowline and the sintering effect.

\subsubsection{Asymmetric structure}

As discussed in Section 3.4, the asymmetric structure is suggested at the outer region of the disk, the positive (bright) structure at R.A. offset $\simeq-0.5$ arcsec and the negative (dark) structure at R.A. offset $\simeq-0.3$ arcsec. Recently Garufi et al. (2020) have observed a bright spiral in the disk of WW Cha by SPHERE observation, which wraps around from east to north in clockwise. In Figure 18, we compare the infrared image given by SPHERE and the image synthesized from the imaginary part of the visibility (the same as that shown in Figure 11). The spiral feature in the SPHERE image has bright and faint parts. The location of the positive asymmetric feature in sub-mm observations coincides with the bright NIR spiral while that of the negative asymmetric feature coincides with the location of faint spiral region of the NIR observation.

In tandem with the radiative transfer modeling, we suggest that the spiral features are formed by gravitational instability. When the value of Toomre's $Q$ is smaller than $\sim 1$, the effects of self-gravity become prominent spiral features appear (e.g., Lodato \& Rice 2004). As shown in Figure 15, hence,

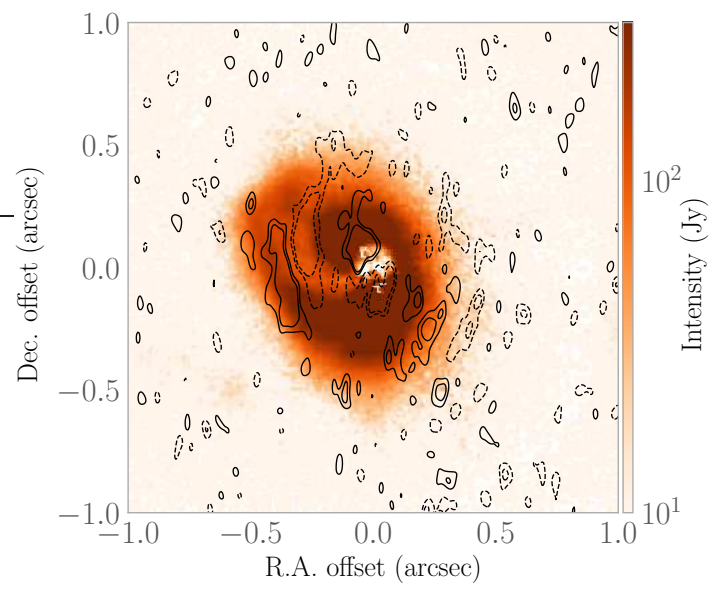

Figure 18. Comparison between the SPHERE image (Garufi et al. 2020) (color) and the residual map for the upper band data shown in Figure 9 (contour). The contours show $\pm 3 \sigma$ and $\pm 5 \sigma$ levels. The solid and dashed contours indicate positive and negative excesses, respectively.

the disk can be gravitational unstable at $R=40-80 \mathrm{au}$, when $\Sigma_{0}=1.5 \mathrm{~g} / \mathrm{cm}^{2}$, if the gas-to-dust ratio is 100 . When the disk was highly gravitationally unstable, we could observe clear spiral waves or fragmented structures. However, we cannot see clear significant spirals or fragments on the disk, except the relatively weak structure at the upper left. Hence, the gas-to-dust ratio may be smaller than 100, especially within the bump region. The faint positive structure at the upper left might be explained by the gravitational instability if the disk is marginally stable with a smaller gasto-dust ratio. Alternatively, we cannot see significant spiral pattern because the disk is optically thick. In this case, the spiral might be observed at longer wavelengths.

Another possible mechanism for making asymmetry is dust concentration in the vortex. Several protoplanetary disks have been observed to have vortex structures, for instance, HD 142527 (Fukagawa et al. 2013; Soon et al. 2019), Sz 91 Tsukagoshi et al. (2014); Canovas et al. (2016), Oph IRS 48 (van der Marel et al. 2015), MWC 758 (Dong et al. 2018b), and etc. One possible mechanism to form such a vortex is trapping dust grains into vortex formed by Rossby wave instability (e.g., Lovelace et al. 1999; Li et al. 2000; Lin 2014; Fu et al. 2014; Ono et al. 2016). However, as different from the above disks, the asymmetric structure found in the disk of WW Cha is so faint that it is not visible in the raw image (Figure 1). It is visible only in the image with subtraction (the right panel of Figure 9 and Figure 11). The region of $R>0.3$ arcsec can be optically thin and the intensity of the asymmetric structure $(\sim 5 \sigma)$ is just $5 \%$ of that of the symmetric structure $(\sim 100 \sigma$ at $R \simeq 0.4$ arcsec, as can be seen in Figure 1). Such a faint structure might be difficult to be formed by the dust trap into the vortex. 
The origin of the negative asymmetric structure could not be explained by the above mechanisms. Further theoretical models are required to discuss the origin of the negative asymmetric structure.

The asymmetric structure may be originated from the collapsing cloud/envelope. The large-scale structure observed by e.g., SPHERE (Garufi et al. 2020) indicate presence of the envelope, but the system is not significantly embedded as the extinction is not so large $\left(A_{V} \sim 4.8 \mathrm{mag}\right.$ (Ribas et al. 2013), compared to e.g., 24 - 36 mag of HL Tau system (ALMA Partnership et al. 2015)). Since the star has a high accretion rate, the asymmetry may be associated to the accretion variability or a jet. So far, we do not see a significant jetlike structures both in the SPHERE and our dust continuum images so it is difficult to address this further with current observations. Further observations (e.g., $\mathrm{H} \alpha$ observations to see accretion variability or jet structure) would be required.

\subsection{Inner cavity and binary}

Although a large cavity is ruled out by the observed image shown in Figure 1, our model allows the existence of a small cavity with the radius of about 1 au. However, further observations are required to confirm the small cavity, because it is much smaller than the angular resolution with the baselines of $<2000 k \lambda$. Moreover, we should note that there is a relatively large uncertainties on the MCMC fitting of the cavity size $\left(R_{\text {cav }}\right)$ and depth $\left(\delta_{\text {cav }}\right)$ as seen in Table 2 . It indicates that our fitting cannot rule out the solution with no cavity.

If the disk has the small cavity, it can be formed by binary interaction (e.g. Artymowicz \& Lubow 1994; Dunhill et al. 2015; Miranda et al. 2017; Thun et al. 2017; Price et al. 2018). The binary separation $a_{\text {bin }}$ may be estimated from the cavity radius $R_{0}$ as $R_{0}=2.5 a_{\text {bin }}$ (Artymowicz \& Lubow 1994), and equivalently $a_{\text {bin }} \simeq 0.4$ au. This separation is roughly consistent with the binary motion observed by Anthonioz et al. (2015).

As shown in Section 3.4, the center of the inner structure can be different in $\sim 2$ mas from the center of the outer structure. When the eccentricity of the binary motion is relatively large, the shape of the cavity induced by the binary interaction is also eccentric (e.g. Thun et al. 2017). On the other hand, the shape of the outer disk can keep a symmetric shape because of the small binary separation. Hence, the binary eccentricity may be relatively large, if the star is binary.

\subsection{Dust size distribution}

Finally, we discuss the size distribution of the dust grains in the disk from the spectral index map, though it is calculated from the narrow range of the observed frequency. As can be seen in the panels (b) and (d) of Figure 1, the spectral index below 2 around the center can be induced by the optical thick scattering emission (Zhu et al. 2019; Liu 2019), and the model with $s_{\text {d,large }}=0.5 \mathrm{~mm}$ can reproduce such a spectral index. In the outer region of $>0.25$ arcsec from the center ( $R>50 \mathrm{au}$ ), the spectral index increases in the outer region, which is consistent with the model with $s_{\mathrm{d} \text {,large }}=1.0 \mathrm{~mm}$. This feature implies that the size of the large dust is larger in the outer disk. Here, we discuss how such a distribution can be realized. When the Stokes number of the dust grains is much smaller than unity, the radial drift velocity can be written by (e.g., Nakagawa et al. 1986)

$$
v_{\mathrm{R}, \mathrm{dust}} \simeq-2 S t \eta V_{R}
$$

where $V_{R}$ denotes the Keplerian rotation velocity, $S t$ is the Stokes number of the dust grains given by $\pi \rho_{d} s_{d} /\left(2 \Sigma_{\text {gas }}\right)$ ( $\rho_{d}$ is the internal density of the dust), and $\eta=d \ln P / d \ln R$ is $\sim 10^{-3}$ in conventional disk models. Our model indicates $\Sigma_{\text {dust }} \sim 1.0 \mathrm{~g} / \mathrm{cm}^{2}$ (Figure 15 ), and hence, the Stokes number of $1 \mathrm{~mm}$-sized dust is about 0.005 when the gas-to-dust ratio is 100 and $\rho_{d}=3$. The radial drift timescale of the grains, $\tau_{\text {drift }}=-R / v_{\mathrm{R} \text {,dust }}$, can be estimated by

$$
\begin{aligned}
\tau_{\text {drift }} \simeq & 5.5 \operatorname{Myr}\left(\frac{\Sigma_{\text {dust }}}{1 \mathrm{~g} / \mathrm{cm}^{2}}\right)\left(\frac{\epsilon}{100}\right)\left(\frac{\rho_{d}}{3 \mathrm{~g} / \mathrm{cm}^{3}}\right)^{-1} \\
& \times\left(\frac{s_{\text {dust }}}{1 \mathrm{~mm}}\right)^{-1}\left(\frac{\eta}{10^{-3}}\right)^{-1}\left(\frac{R}{50 \mathrm{au}}\right)^{3 / 2}
\end{aligned}
$$

where $\epsilon$ is the gas-to-dust ratio and $s_{\text {dust }}$ is the size of the dust grain, respectively. WW Cha is young and the age is about $\lesssim 1 \mathrm{Myr}$, which is shorter than the drift timescale of $1 \mathrm{~mm}-$ sized dust and longer than the growth timescale of the dust, $\tau_{\text {growth }} \simeq \epsilon / \Omega_{\mathrm{K}}=0.005 \operatorname{Myr}(50 \mathrm{au} / R)^{3 / 2}$ with $\epsilon=100$ (Brauer et al. 2008). Hence, the $1 \mathrm{~mm}$-sized dust grains observed in the outer region is consistent with the dust drift. In the inner region, the drift timescale of the dust grains becomes shorter. Considering the star is as young as $<1 \mathrm{Myr}$, the drift timescale can be comparable with the stellar age at $R<16$ au. This may explain the reason why the size of the dust grains is smaller than in the outer region.

The size of the grains can be determined by turbulent fragmentation (Birnstiel et al. 2010). In this case, the $\alpha$ parameter relevant to that the fragmentation threshold size equals to $\sim 1 \mathrm{~mm}$ is

$$
\begin{aligned}
\alpha & =1.57 \times 10^{-2}\left(\frac{\Sigma_{\text {dust }}}{1 \mathrm{~g} / \mathrm{cm}^{2}}\right)\left(\frac{\epsilon}{100}\right) \\
& \times\left(\frac{\rho_{d}}{1 \mathrm{~g} / \mathrm{cm}^{3}}\right)^{-1}\left(\frac{s_{\text {dust }}}{1 \mathrm{~mm}}\right)^{-1}\left(\frac{v_{f}}{10 \mathrm{~m} / \mathrm{s}}\right)^{2}\left(\frac{T}{50 \mathrm{~K}}\right)^{-1},
\end{aligned}
$$

where $v_{f}$ is the fragmentation threshold velocity. Hence, considering the mid-plane temperature shown in Figure 15, we can estimate $\alpha \simeq 10^{-2}$. This relatively large $\alpha$ is consistent with the high accretion rate onto the star (Manara et al. 
2016), whereas it is larger than a value of $\alpha$ estimated by the recent observations of the disks around the aged stars, namely, HD 163296 (Flaherty et al. 2015), and TW Hya (Teague et al. 2016; Flaherty et al. 2018), namely, $\alpha \lesssim 10^{-3}$. Such a high viscosity may be due to turbulence induced by gravitational instability (e.g., Boley et al. 2006).

As mentioned in Section 4.2.3, we note that the above discussion is based on the spectral index provided only from the very narrow range within band 6 . The spectral index should be investigated by future multi-wavelength observations.

\section{CONCLUSION}

We presented the dust continuum emission of the protoplanetary disk around WW Cha, observed by ALMA band 6 . Our conclusions are summarized as follows:

1. The dust continuum image clearly shows no large cavity, and a faint dust bump (Figure 1). We also found the asymmetric structure at the center (Figure 2). Moreover, since the visibility is clearly different in the upper $(233.0 \mathrm{GHz})$ and lower bands (216.7 GHz) (Figure 4), we can obtain the spectral index map. The spectral index around the center is below 2 , and it becomes larger in the outer region.

2. We constructed a model to fit the observation in visibility domain by MCMC fitting (Section 3). Our model has a bump extending from $\sim 40$ au from the central star to $\sim 80 \mathrm{au}$, with two local peaks located at $\sim 40$ au and $\sim 70-$ au. As a result of radiative transfer simulations (Figure 15), the midplane temperature around the outer peak is close to the freezeout temperature of $\mathrm{CO}$ on water ice $(\sim 30 \mathrm{~K})$. The midplane temperature around the inner peak is about $50 \mathrm{~K}$, which is close to the condensation temperature of $\mathrm{H}_{2} \mathrm{~S}$ and it is also close to the temperature that the sintering can be caused for several species. Hence, this bump may be induced by the snowline and the sintering effect.

3. The residual map between the observed data and best fit model indicates asymmetric structures at the center and the upper left of the disk. We also confirmed those asymmetric structures by a model-independent method, which is imaging of the imaginary part of the visibility of the observed data (Section 3.4). These structure could be robust, though the amplitude of the asymmetric structures are faint $(\sim 5 \sigma$ level $)$ as compared with the symmetric structure.

4. The spectral index map given by the observation may be consistent with the result of radiative transfer simulations with the relatively large dust grains $(1 \mathrm{~mm})$ in the outer region, whereas the result of the simulation with the smaller dust grains $(0.5 \mathrm{~mm})$ can be suitable for the region close to the center (Figures 16 and 17). This implies that the size of the dust grains is larger than that in the inner region. As discussed in Section 5.3, such a distribution is consistent with the radial drift and collisional growth of the dust grains, because of the massive disk around a young star.

We thank Ryo Tazaki for providing the optical constant data to calculate opacity of dust grains. KDK was also supported by JSPS Core-to-Core Program "International Network of Planetary Sciences" and JSPS KAKENHI. This work is in part supported by JSPS KAKENHI grant Nos. 18H05441 and 17H01103. Y.H. is supported by the Jet Propulsion Laboratory, California Institute of Technology, under a contract with the National Aeronautics and Space. H.B.L. is supported by the Ministry of Science and Technology (MoST) of Taiwan (grant Nos. 108-2112-M-001002-MY3). This paper makes use of the following ALMA data: ADS/JAO.ALMA\#2017.1.00286.S. ALMA is a partnership of ESO (representing its member states), NSF (U.S.), and NINS (Japan), together with NRC (Canada), NSC and ASIAA (Taiwan), and KASI (Republic of Korea), in cooperation with the Republic of Chile. The joint ALMA observatory is operated by ESO, auI/NRAO, and NAOJ. Data analysis was carried out on the Multi-wavelength Data Analysis System operated by the Astronomy Data Center (ADC), National Astronomical Observatory of Japan. Radiative transfer simulations were carried out on analysis servers at Center for Computational Astrophysics, National Astronomical Observatory of Japan.

Software: $\quad$ RADMC-3D (Dullemond \& Dominik 2005), CASA v5.6 (McMullin et al. 2007), vis_sample https://github.com/AstroChem/vis_sample, emcee (Foreman-Mackey et al. 2013), Matplotlib (Hunter 2007, http://matplotlib.org), NumPy (van der Walt et al. 2011, http://www.numpy.org)

\section{APPENDIX}

\section{A. STATISTICS OF VISIBILITY DATA}

In this appendix, we show the statistics of the visibility data. In the upper panel of Figure 19, we shows the real parts of the visibility for the C43-4 (compact configuration) and C43-8 (sparse configuration) data in the upper and lower bands, separately. 

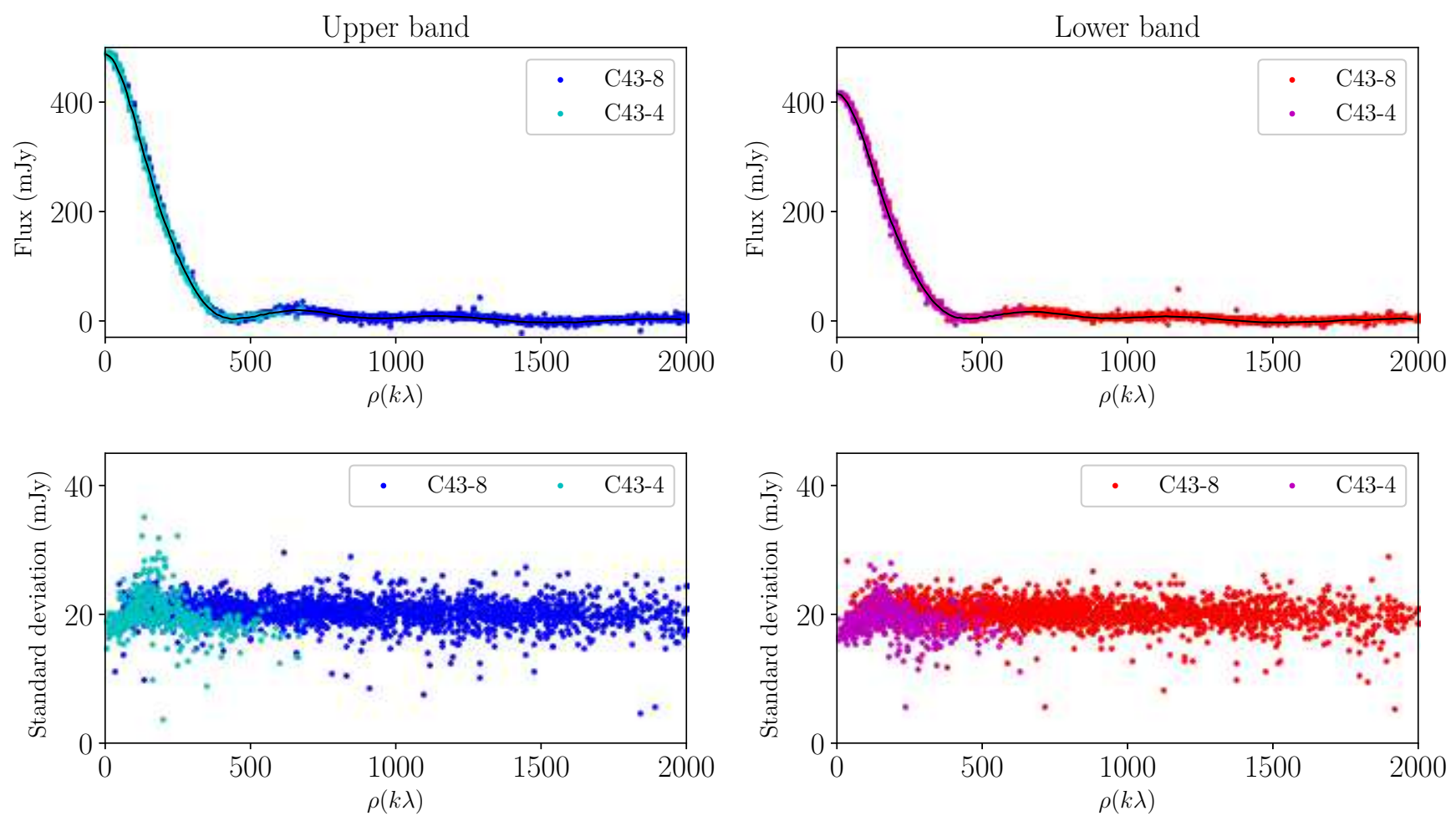

Figure 19. (Upper) Real parts of the visibility of the C43-4 (compact array configuration) and C43-8 (sparse array configuration) data in the upper and lower bands. The visibilities are averaged within the bin which the radial width is the same as that described in Section 2 and the azimuthal width is $0.2 \pi$. The thin solid lines indicates the visibilities shown in Figure 4. (Lower) Standard deviation of the data within the bin.

The visibility in the figure is averaged the bin of the same $u v$-distance width as Figure 4 but with the azimuthal width of $0.2 \pi$, instead of whole $2 \pi$ in Figure 4. Hence, the figure enable us to see the scatter of data in the azimuthal direction. For reference, we plot the visibility shown in Figure 4. In the lower panel of Figure 19, we shows the standard deviation of the data within the bin. The visibilities of the C43-4 and C43-8 data are similar to each other. The standard deviations are similar in all the data, namely $\sigma \simeq 20 \mathrm{mJy}$. However, around $\rho=200 k \lambda$, there are some points with larger standard deviations in the C43-4 data (especially at the upper band data). Because of this data scatter, the value of $\chi^{2}$ increases around $\rho=200 k \lambda$ when the visibility combined by the short and long baseline data for the MCMC fitting.

Figure 20 is the same as Figure 19 but for the imaginary part of the visibility. The standard deviation of the imaginary part is larger than that of the real part in the shorter baseline, namely $\rho \lesssim 200 k \lambda$, whereas it is comparable with that of the real parts at long baseline. Hence, the imaginary part of the visibility shown in Figure 10 has a relatively large error at short baseline.

\section{B. POSTERIORS OF MCMC FITTING}

The posterior of the MCMC fitting for the upper hand data is shown in Figure 21 and that for the lower band data is shown in Figure 22.

\section{SPECTRAL INDEX MAP WITH A POWER-LAW SIZE DISTRIBUTION FOR LARGE DUST GRAINS}

In Section 4, we carried out radiative transfer simulations with large grains which has a Gaussian size distribution around the specific radius of the dust grains. Here, we show the results of radiative transfer simulations with large grains which has a powerlaw size distribution like that of the small grains, and investigate the dependence of the dust size distribution on the spectral index map.

As in the cases described in Section 4, we consider two-kind of dust grains: one represents small grains, other is large grains. The size distribution of the small dust is the same as that described in Section 4. For the large grains, the number density of the dust grains is proportional to $s^{p}$, and we adopted $p=-3.5$ and -2.5 cases. The minimum size of the large grains is $0.1 \mathrm{~mm}$, and we consider three maximum sizes of the large grains, namely, $s_{\max }=1 \mathrm{~mm}$ and $0.5 \mathrm{~mm}$. The stellar luminosity is $22 L_{\odot}$ and $\Sigma_{0}=1.5 \mathrm{~g} / \mathrm{cm}^{2}$, and other parameters are the same these described in Section 4. 

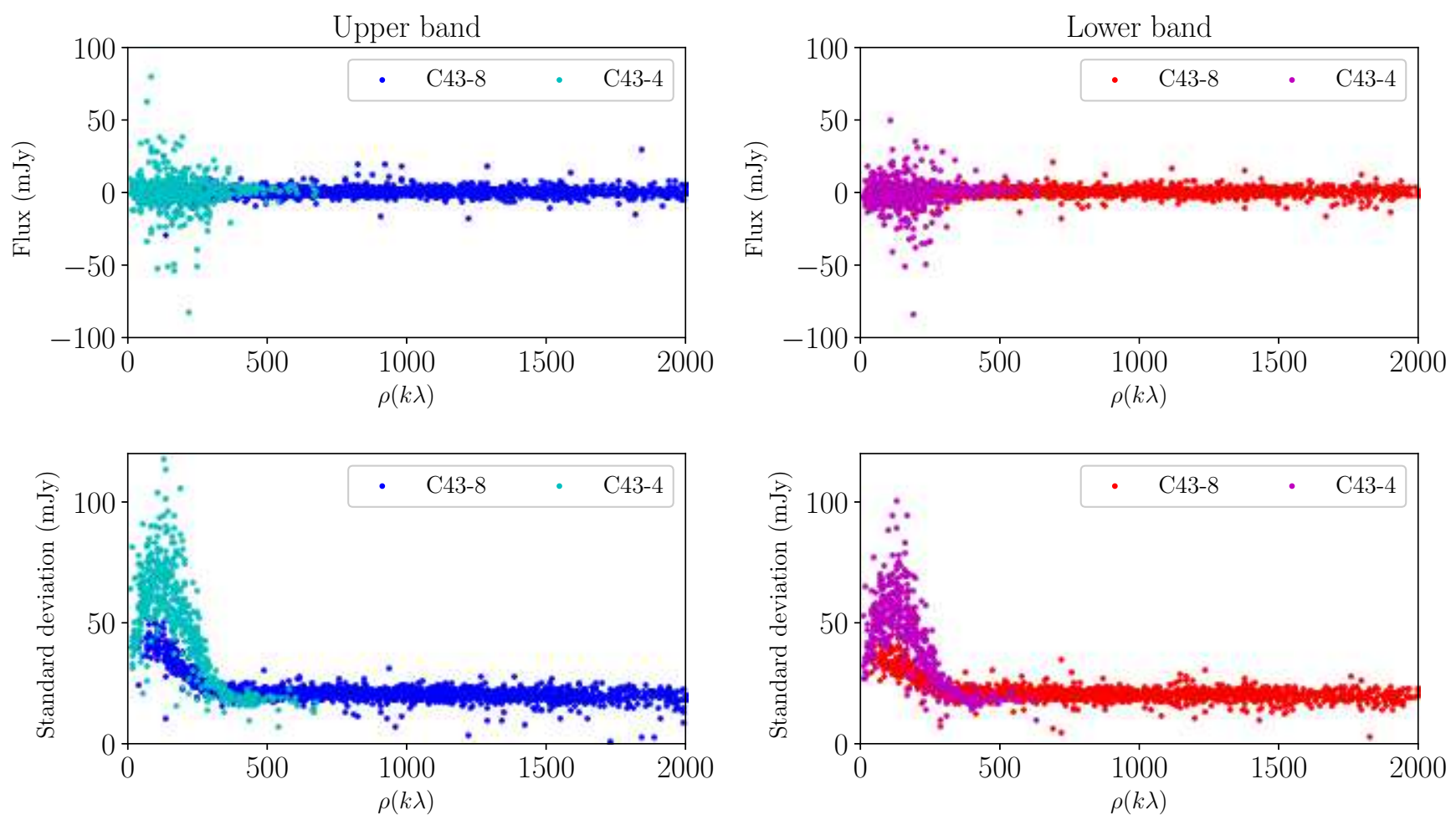

Figure 20. The same as Figure 19, but for the imaginary parts.

Figure 23 shows the spectral index along the major axis for the cases with $p=-3.5$ and $p=-2.5$. In all the cases, the spectral index is about 2 (but slightly larger than 2), and it increases in outer region.

\section{SPECTRAL INDEX MAP FOR ALMA BANDS}

For future observations, in this appendix, we present a few examples of the spectral index map given by radiative transfer simulations. As in the previous section, $L_{\odot}=22 L_{\odot}$ and $\Sigma_{0}=1.5 \mathrm{~g} / \mathrm{cm}^{2}$, and other parameters are the same these described in Section 4. As different from these shown in Section 4, the spectral index presented in this section is not calculated through the tclean task. The spectral index is calculated by just the differences of the fluxes convoluted with a Gaussian filter with 0.1 arcsec standard deviation. We calculated the spectral index as the difference of the fluxes among ALMA band 3, band 6, band 7 , and band 9 .

Figure 24 shows the spectral index along the major axis, when the large grains has a Gaussian (log-normal) distribution (for the detail, see Section 4). The spectral index depends on the choice of the bands used. The spectral index calculated from bands with longer wavelengths becomes larger, as the opacity is smaller and it is optically thin. For instance, the spectral index calculated from the band 3 and band 6 fluxes are larger than the spectral index calculated from other pairs of bands. The spectral index calculated from the band 7 and band 9 is below 2 around the center, regardless of $s_{\mathrm{d} \text {,large }}$. It is worth pointing out that the spectral index calculated from band 6 and band 7 significantly different from that calculated from the upper and lower bands of band 6 , in the cases with $s_{\mathrm{d} \text {,large }}=0.5 \mathrm{~mm}$. Figure 25 shows the same as that shown in Figure 25, but for the cases that the large dust grains has a power-law distribution of $\propto s^{-3.5}$. The distributions of the spectral index calculated by band 6 and 7 is similar to that in the case with the Gaussian distribution shown in Figure 24, which is below 2 around the center. On the other hand, the spectral index calculated from the band 3 and 6 is larger than that in the case with the power-law distributions, as compared with that in the cases with the Gaussian distribution. We may be able to constrain the dust size distribution from the difference of the spectral indexes calculated from the different pair of the bands.

\section{REFERENCES}

Akiyama, E., Muto, T., Kusakabe, N., et al. 2015, ApJL, 802, L17, doi: 10.1088/2041-8205/802/2/L17
Akiyama, E., Hashimoto, J., Liu, H. B., et al. 2016, AJ, 152, 222, doi: 10.3847/1538-3881/152/6/222 


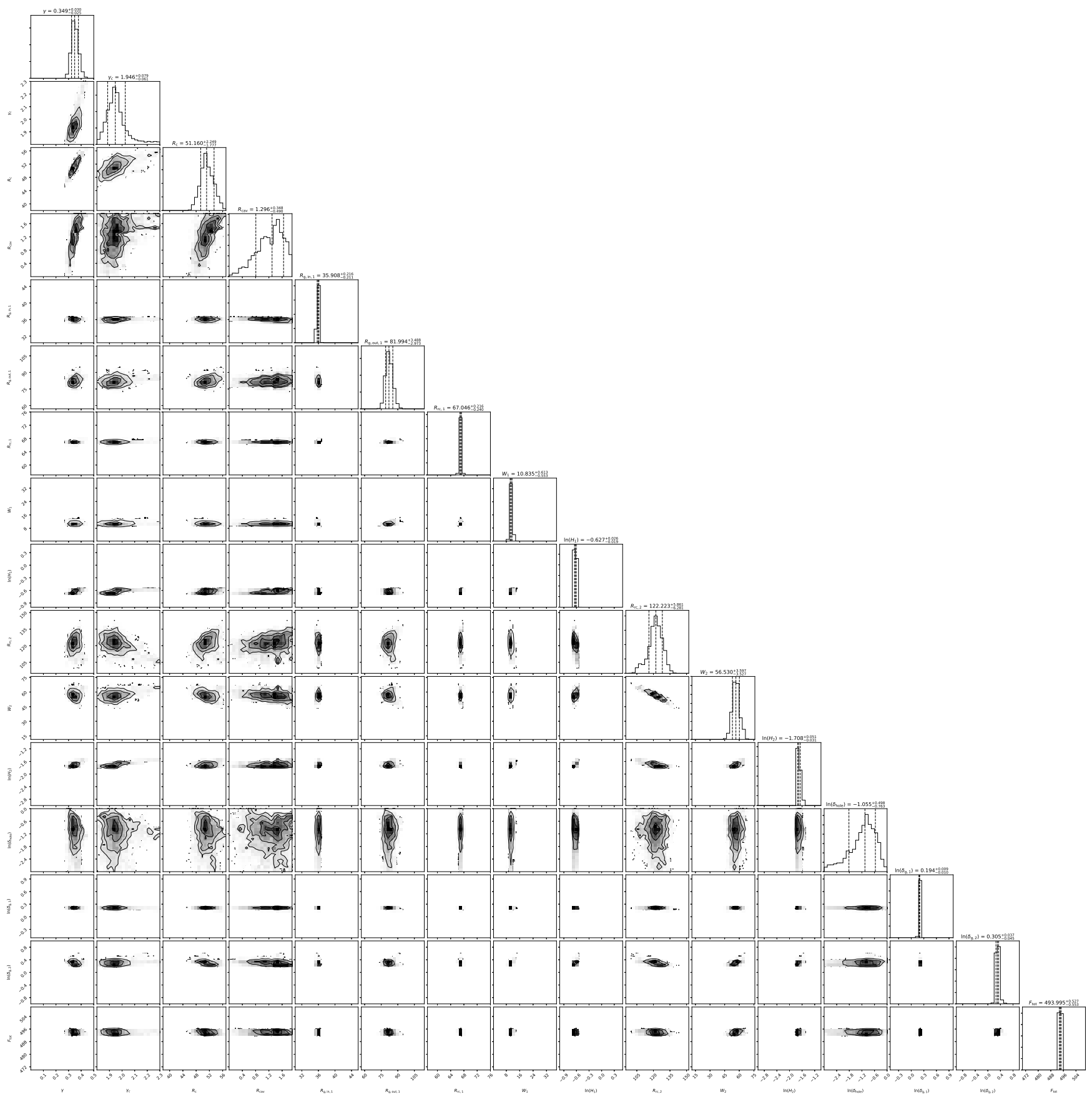

Figure 21. Posterior of the MCMC fitting for the upper band data.

ALMA Partnership, Brogan, C. L., Pérez, L. M., et al. 2015, ApJL, 808, L3, doi: 10.1088/2041-8205/808/1/L3

Andrews, S. M., Huang, J., Pérez, L. M., et al. 2018, ApJ, 869, L41, doi: 10.3847/2041-8213/aaf741

Anthonioz, F., Ménard, F., Pinte, C., et al. 2015, A\&A, 574, A41, doi: 10.1051/0004-6361/201424520

Artymowicz, P., \& Lubow, S. H. 1994, ApJ, 421, 651, doi: $10.1086 / 173679$
Birnstiel, T., Dullemond, C. P., \& Brauer, F. 2010, A\&A, 513, A79, doi: 10.1051/0004-6361/200913731

Birnstiel, T., Dullemond, C. P., Zhu, Z., et al. 2018, ApJL, 869, L45, doi: 10.3847/2041-8213/aaf743

Boley, A. C., Mejía, A. C., Durisen, R. H., et al. 2006, ApJ, 651, 517, doi: $10.1086 / 507478$

Brauer, F., Dullemond, C. P., \& Henning, T. 2008, A\&A, 480, 859, doi: 10.1051/0004-6361:20077759 


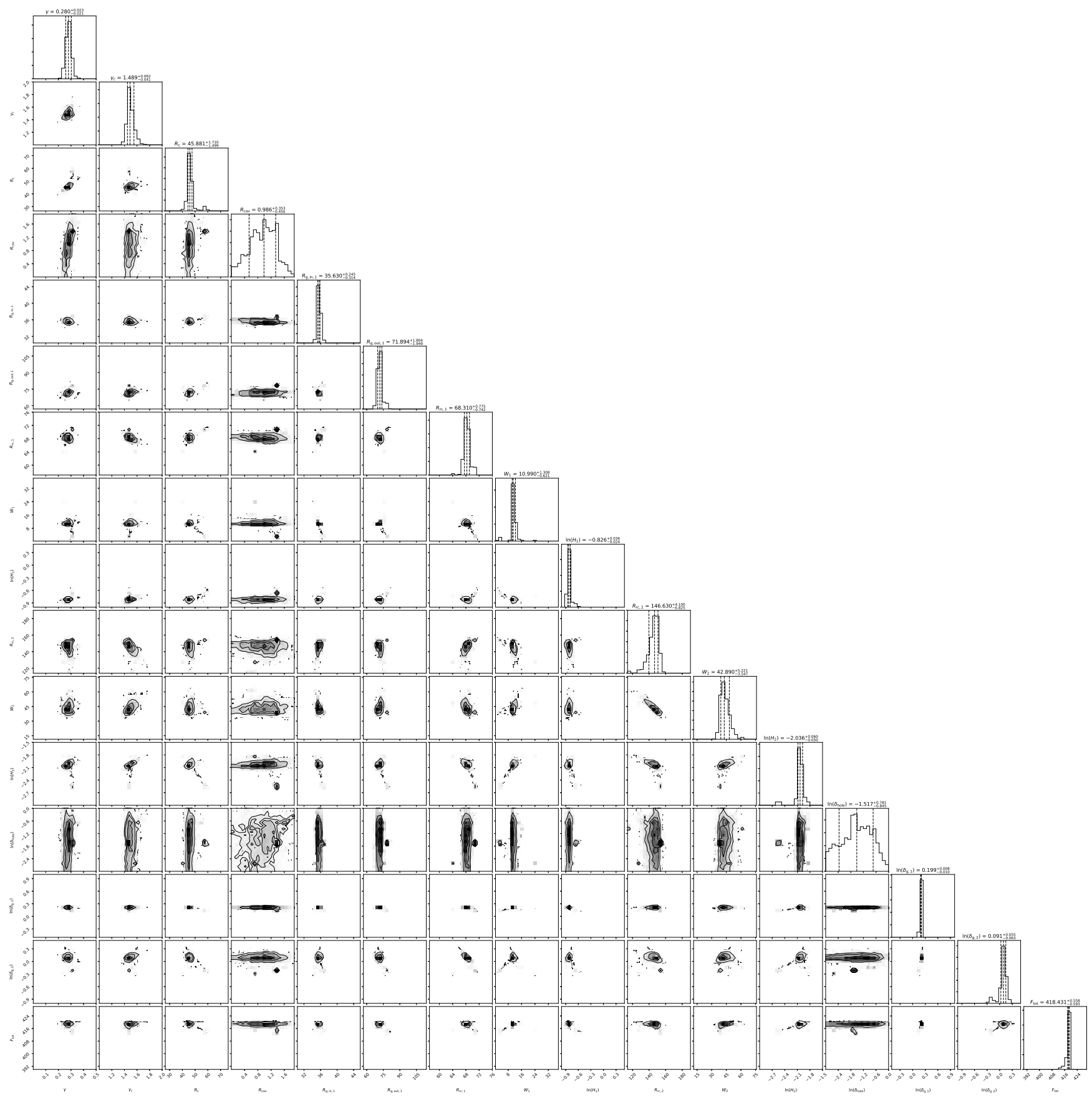

Figure 22. Posterior of the MCMC fitting for the lower band data.

Canovas, H., Caceres, C., Schreiber, M. R., et al. 2016, MNRAS, 458, L29, doi: 10.1093/mnrasl/slw006

Chiang, E. I., \& Goldreich, P. 1997, ApJ, 490, 368, doi: $10.1086 / 304869$

Cieza, L. A., Casassus, S., Pérez, S., et al. 2017, ApJL, 851, L23, doi: 10.3847/2041-8213/aa9b7b

Cutri, R. M., \& et al. 2014, VizieR Online Data Catalog, II/328

Dong, R., Najita, J. R., \& Brittain, S. 2018a, ApJ, 862, 103, doi: 10.3847/1538-4357/aaccfc
Dong, R., Zhu, Z., \& Whitney, B. 2015, ApJ, 809, 93, doi: 10.1088/0004-637X/809/1/93

Dong, R., Liu, S.-y., Eisner, J., et al. 2018b, ApJ, 860, 124, doi: 10.3847/1538-4357/aac6cb

Dullemond, C. P., \& Dominik, C. 2005, A\&A, 434, 971, doi: 10.1051/0004-6361:20042080

Dullemond, C. P., Juhasz, A., Pohl, A., et al. 2012, RADMC-3D: A multi-purpose radiative transfer tool. http://ascl.net/1202.015 


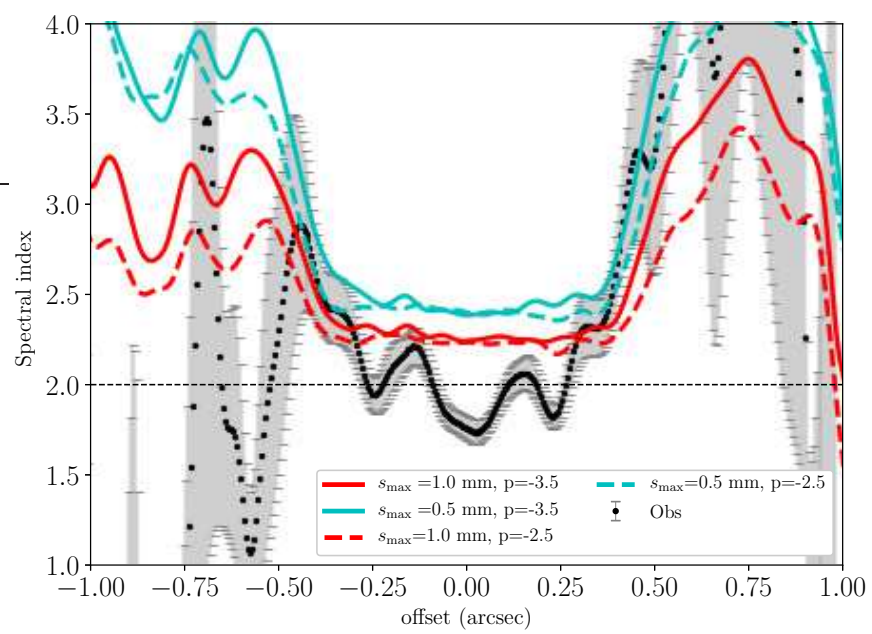

Figure 23. The same as Figure 17, but for the power-law sized large grains, and in the case of $p=-3.5$ (solid lines) and the case with $p=-2.5$ (dashed lines).
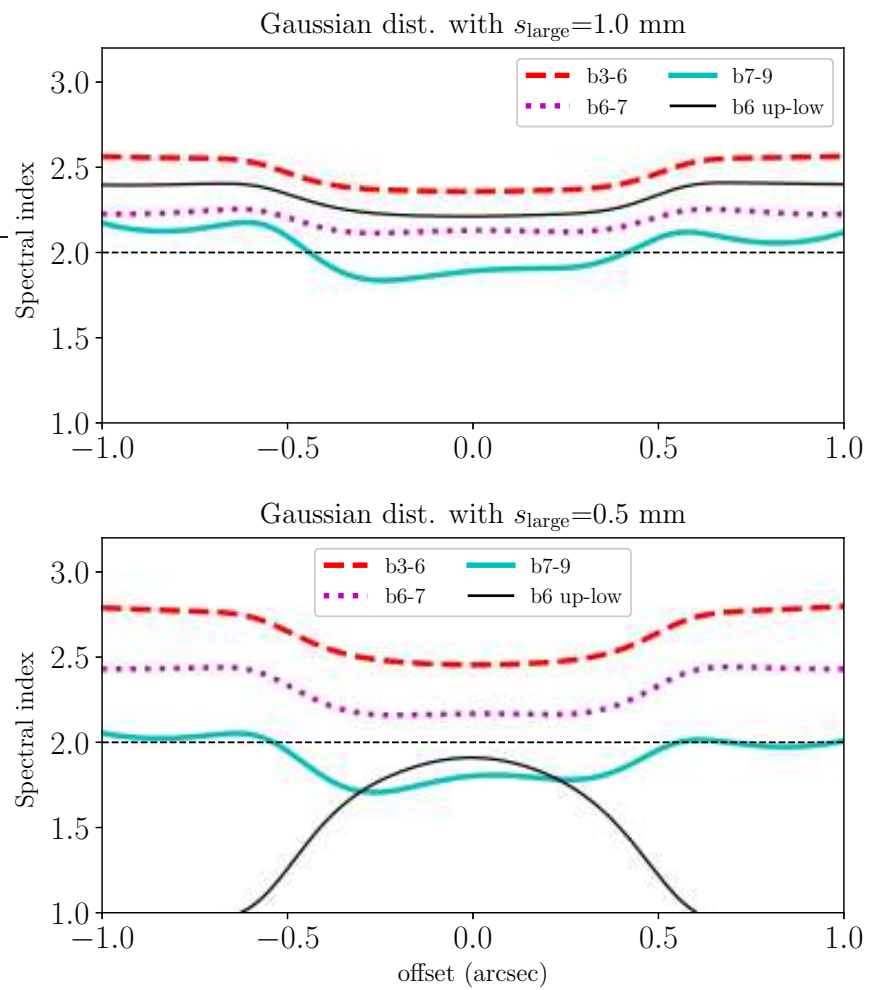

Figure 24. The spectral index distributions along the major axis in the cases that the large dust grains have a Gaussian size distribution with $s_{\mathrm{d} \text {,large }}=1 \mathrm{~mm}$ (top), $0.5 \mathrm{~mm}$ (bottom). The dashed, dotted, and solid lines indicate the spectral indices calculated from the fluxes at band 3 $(3.1 \mathrm{~mm})$ and at band $6(1.3 \mathrm{~mm})$, the fluxes at band 6 and at band $7(0.87 \mathrm{~mm})$, and the fluxes at band 7 and band $9(0.45 \mathrm{~mm})$, respectively. The thin solid line indicates the spectral index given by the upper and lower bands of band 6 as the same as presented in the main text, for reference. 

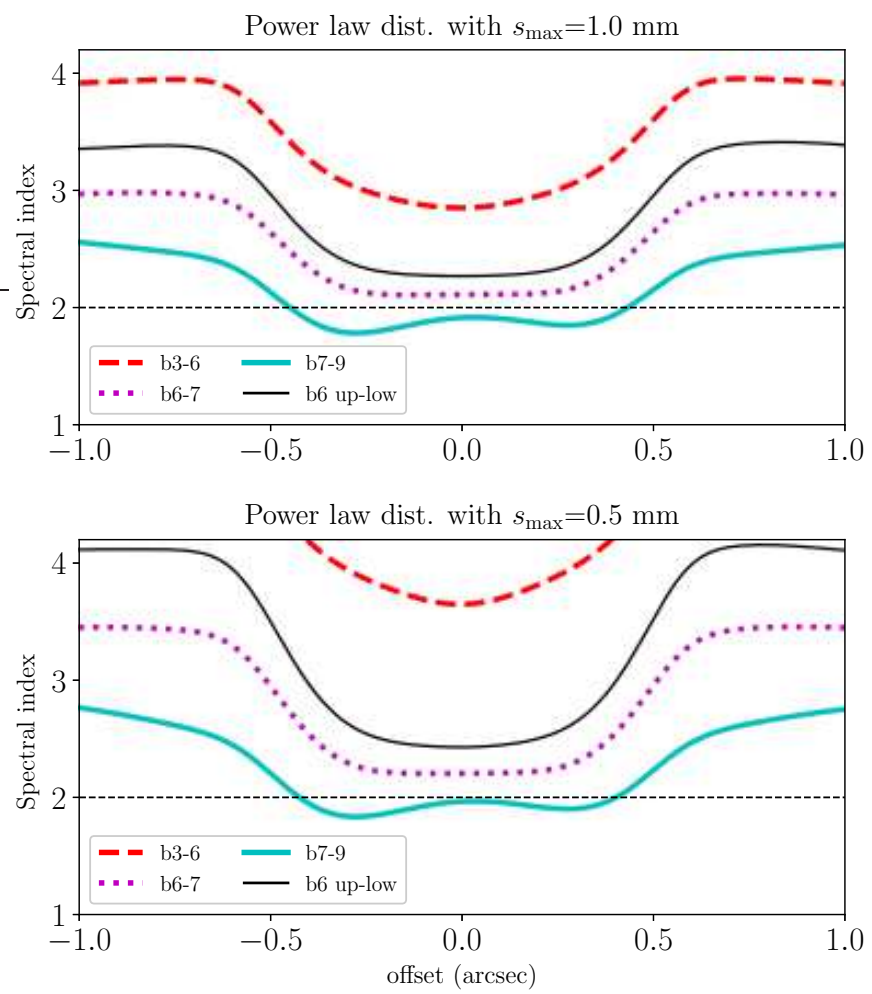

Figure 25. The same as Figure 24, but the large grains have a power-law size distribution. 
Dullemond, C. P., Birnstiel, T., Huang, J., et al. 2018, ApJL, 869, L46, doi: 10.3847/2041-8213/aaf742

Dunhill, A. C., Cuadra, J., \& Dougados, C. 2015, MNRAS, 448, 3545, doi: 10.1093/mnras/stv284

Espaillat, C., Furlan, E., D’Alessio, P., et al. 2011, ApJ, 728, 49, doi: 10.1088/0004-637X/728/1/49

Facchini, S., Benisty, M., Bae, J., et al. 2020, A\&A, 639, A121, doi: 10.1051/0004-6361/202038027

Flaherty, K. M., Hughes, A. M., Rosenfeld, K. A., et al. 2015, ApJ, 813, 99, doi: 10.1088/0004-637X/813/2/99

Flaherty, K. M., Hughes, A. M., Teague, R., et al. 2018, ApJ, 856, 117, doi: 10.3847/1538-4357/aab615

Follette, K. B., Grady, C. A., Swearingen, J. R., et al. 2015, ApJ, 798, 132, doi: 10.1088/0004-637X/798/2/132

Foreman-Mackey, D., Hogg, D. W., Lang, D., \& Goodman, J. 2013, PASP, 125, 306, doi: 10.1086/670067

Fu, W., Li, H., Lubow, S., Li, S., \& Liang, E. 2014, ApJL, 795, L39, doi: 10.1088/2041-8205/795/2/L39

Fukagawa, M., Tsukagoshi, T., Momose, M., et al. 2013, PASJ, 65, L14, doi: 10.1093/pasj/65.6.L14

Gaia Collaboration, Brown, A. G. A., Vallenari, A., et al. 2020, arXiv e-prints, arXiv:2012.01533.

https://arxiv.org/abs/2012.01533

—. 2018, A\&A, 616, A1, doi: 10.1051/0004-6361/201833051

Garufi, A., Avenhaus, H., Pérez, S., et al. 2020, A\&A, 633, A82, doi: 10.1051/0004-6361/201936946

Gutermuth, R. A., Megeath, S. T., Myers, P. C., et al. 2009, ApJS, 184, 18, doi: 10.1088/0067-0049/184/1/18

Huang, J., Andrews, S. M., Dullemond, C. P., et al. 2018, ApJL, 869, L42, doi: 10.3847/2041-8213/aaf740

Hunter, J. D. 2007, Computing in Science and Engineering, 9, 90, doi: 10.1109/MCSE.2007.55

Ishihara, D., Onaka, T., Kataza, H., et al. 2010, A\&A, 514, A1, doi: 10.1051/0004-6361/200913811

Kanagawa, K. D., Muto, T., Okuzumi, S., et al. 2018, ApJ, 868, 48, doi: 10.3847/1538-4357/aae837

Kim, S., Takahashi, S., Nomura, H., et al. 2020, ApJ, 888, 72, doi: 10.3847/1538-4357/ab5d2b

Li, H., Finn, J. M., Lovelace, R. V. E., \& Colgate, S. A. 2000, ApJ, 533, 1023, doi: 10.1086/308693

Lin, M.-K. 2014, MNRAS, 437, 575, doi: 10.1093/mnras/stt1909

Liu, H. B. 2019, ApJL, 877, L22, doi: 10.3847/2041-8213/ab1f8e

Lodato, G., \& Rice, W. K. M. 2004, MNRAS, 351, 630, doi: 10.1111/j.1365-2966.2004.07811.x

Lommen, D., Maddison, S. T., Wright, C. M., et al. 2009, A\&A, 495, 869, doi: 10.1051/0004-6361:200810999

Lommen, D., Wright, C. M., Maddison, S. T., et al. 2007, A\&A, 462, 211, doi: 10.1051/0004-6361:20066255

Long, F., Pinilla, P., Herczeg, G. J., et al. 2018, ApJ, 869, 17, doi: 10.3847/1538-4357/aae8e1
Loomis, R. A., Öberg, K. I., Andrews, S. M., \& MacGregor, M. A. 2017, ApJ, 840, 23, doi: 10.3847/1538-4357/aa6c63

Lovelace, R. V. E., Li, H., Colgate, S. A., \& Nelson, A. F. 1999, ApJ, 513, 805, doi: 10.1086/306900

Luhman, K. L. 2007, ApJS, 173, 104, doi: 10.1086/520114

Macías, E., Anglada, G., Osorio, M., et al. 2017, ApJ, 838, 97, doi: 10.3847/1538-4357/aa6620

Manara, C. F., Fedele, D., Herczeg, G. J., \& Teixeira, P. S. 2016, A\&A, 585, A136, doi: 10.1051/0004-6361/201527224

McMullin, J. P., Waters, B., Schiebel, D., Young, W., \& Golap, K. 2007, in Astronomical Society of the Pacific Conference Series, Vol. 376, Astronomical Data Analysis Software and Systems XVI, ed. R. A. Shaw, F. Hill, \& D. J. Bell, 127

Miranda, R., Muñoz, D. J., \& Lai, D. 2017, MNRAS, 466, 1170, doi: 10.1093/mnras/stw3189

Momose, M., Morita, A., Fukagawa, M., et al. 2015, PASJ, 67, 83, doi: 10.1093/pasj/psv051

Muto, T., \& Inutsuka, S.-i. 2009, ApJ, 695, 1132, doi: 10.1088/0004-637X/695/2/1132

Nakagawa, Y., Sekiya, M., \& Hayashi, C. 1986, Icarus, 67, 375, doi: 10.1016/0019-1035(86)90121-1

Okuzumi, S., Momose, M., Sirono, S.-i., Kobayashi, H., \& Tanaka, H. 2016, ApJ, 821, 82, doi: 10.3847/0004-637X/821/2/82

Ono, T., Muto, T., Takeuchi, T., \& Nomura, H. 2016, ApJ, 823, 84, doi: 10.3847/0004-637X/823/2/84

Paardekooper, S.-J., \& Mellema, G. 2004, A\&A, 425, L9, doi: 10.1051/0004-6361:200400053

Pascucci, I., Testi, L., Herczeg, G. J., et al. 2016, ApJ, 831, 125, doi: 10.3847/0004-637X/831/2/125

Pinilla, P., Birnstiel, T., Ricci, L., et al. 2012, A\&A, 538, A114, doi: 10.1051/0004-6361/201118204

Pinilla, P., de Juan Ovelar, M., Ataiee, S., et al. 2015, A\&A, 573, A9, doi: 10.1051/0004-6361/201424679

Price, D. J., Cuello, N., Pinte, C., et al. 2018, MNRAS, 477, 1270, doi: $10.1093 / \mathrm{mnras} / \mathrm{sty} 647$

Rau, U., \& Cornwell, T. J. 2011, A\&A, 532, A71, doi: 10.1051/0004-6361/201117104

Ribas, Á., Merín, B., Bouy, H., et al. 2013, A\&A, 552, A115, doi: 10.1051/0004-6361/201220960

Ribas, Á., Espaillat, C. C., Macías, E., et al. 2017, ApJ, 849, 63, doi: 10.3847/1538-4357/aa8e99

Rodmann, J., Henning, T., Chandler, C. J., Mundy, L. G., \& Wilner, D. J. 2006, A\&A, 446, 211, doi: 10.1051/0004-6361:20054038

Soon, K.-L., Momose, M., Muto, T., et al. 2019, PASJ, 71, 124, doi: 10.1093/pasj/psz112

Takahashi, S. Z., \& Inutsuka, S.-i. 2014, ApJ, 794, 55, doi: 10.1088/0004-637X/794/1/55

—. 2016, AJ, 152, 184, doi: 10.3847/0004-6256/152/6/184 
Teague, R., Guilloteau, S., Semenov, D., et al. 2016, A\&A, 592, A49, doi: 10.1051/0004-6361/201628550

Thun, D., Kley, W., \& Picogna, G. 2017, A\&A, 604, A102, doi: 10.1051/0004-6361/201730666

Tominaga, R. T., Inutsuka, S.-i., \& Takahashi, S. Z. 2018, PASJ, 70, 3, doi: 10.1093/pasj/psx143

Toomre, A. 1964, ApJ, 139, 1217, doi: 10.1086/147861

Tsukagoshi, T., Momose, M., Hashimoto, J., et al. 2014, ApJ, 783, 90, doi: 10.1088/0004-637X/783/2/90

van der Marel, N., Dong, R., di Francesco, J., Williams, J. P., \& Tobin, J. 2019, ApJ, 872, 112, doi: 10.3847/1538-4357/aafd31

van der Marel, N., Pinilla, P., Tobin, J., et al. 2015, ApJL, 810, L7, doi: 10.1088/2041-8205/810/1/L7

van der Marel, N., Verhaar, B. W., van Terwisga, S., et al. 2016, A\&A, 592, A126, doi: 10.1051/0004-6361/201628075 van der Marel, N., Williams, J. P., \& Bruderer, S. 2018, ApJL, 867, L14, doi: 10.3847/2041-8213/aae88e

van der Walt, S., Colbert, S. C., \& Varoquaux, G. 2011, Computing in Science and Engineering, 13, 22, doi: 10.1109/MCSE.2011.37

Vorobyov, E. I., Elbakyan, V. G., Takami, M., \& Liu, H. B. 2020, A\&A, 643, A13, doi: 10.1051/0004-6361/202038122

Zhang, K., Blake, G. A., \& Bergin, E. A. 2015, ApJL, 806, L7, doi: 10.1088/2041-8205/806/1/L7

Zhu, Z., Nelson, R. P., Dong, R., Espaillat, C., \& Hartmann, L. 2012, ApJ, 755, 6, doi: 10.1088/0004-637X/755/1/6

Zhu, Z., Zhang, S., Jiang, Y.-F., et al. 2019, ApJL, 877, L18, doi: 10.3847/2041-8213/ab1f8c 